\title{
A CONTRIBUTION TO KNOWLEDGE OF THE BALKAN LEPIDOPTERA. SOME PYRALOIDEA (LEPIDOPTERA: CRAMBIDAE \& PYRALIDAE) ENCOUNTERED RECENTLY IN SOUTHERN SERBIA, MONTENEGRO, THE REPUBLIC OF MACEDONIA AND ALBANIA
}

\author{
COLIN W. PLANT ${ }^{*}$, STOYAN BESHKOV ${ }^{2}$, PREDRAG JAKŠIĆ ${ }^{3}$, ANA NAHIRNIĆ \\ ${ }^{1} 14$ West Road, Bishops Stortford, Hertfordshire, CM23 3QP, England \\ ${ }^{2}$ National Museum of Natural History, Sofia, Bulgaria \\ ${ }^{3}$ Faculty of Natural Science and Mathematics, University of Priština, Kosovska Mitrovica, Serbia
}

\begin{abstract}
Pyraloidea (Lepidoptera: Crambidae \& Pyralidae) were sampled in the territories of southern Serbia, Montenegro, the Former Yugoslav Republic of Macedonia and Albania on a total of 53 occasions during 2014, 2016 and 2017. A total of 173 species is reported here, comprising 97 Crambidae and 76 Pyralidae. Based upon published data, 29 species appear to be new to the fauna of Serbia, 5 species are new to the fauna of Macedonia and 37 are new to the fauna of Albania. The data are discussed.
\end{abstract}

Keywords: Faunistics, Serbia, Montenegro, Republic of Macedonia, Albania, Pyraloidea, Pyralidae, Crambidae.

\section{INTRODUCTION}

Pyraloidea (Lepidoptera: Crambidae and Pyralidae) have been examined in detail in the neighbouring territory of the Republic of Bulgaria and the results have been published by one of us (Plant, 2016). That work presented data for the 386 species and 3 additional subspecies known from that country. The bulk of modern data used in that review originated from extensive field surveys undertaken by CWP during the period from 2002 to 2015, but also of importance was additional contemporary information obtained from ongoing Lepidoptera field surveys by SB and Dr Boyan Zlatkov and passed to CWP for examination. During the course of their field work, Beshkov and Zlatkov also made occasional forays across the border into Serbia, Montenegro, Macedonia and Albania and pyraloid material from those trips also found its way to CWP in England. Examination of the literature suggests that very little is published or known about the "pyrales" of this region of the southern Balkans and so the ongoing sampling by SB and AN was supplemented by an expedition during 2017 to target these moths in particular.

\section{METHODOLOGY}

De facto sampling in 2014 and 2016

During the years 2014 and 2016, several privately-funded expeditions were undertaken by $\mathrm{SB}$ and $\mathrm{AN}$ in order to sample Noctuoidea and other "macro" moths in the territory of southern Serbia, Montenegro, Macedonia and Albania, using various types

* Corresponding author: cpauk1@ntlworld.com BIOLOGY of light trap. Some sites were visited on more than one occasion; others were sampled once only.

As a by-product of this work, all remaining material from the traps was returned to Sofia where Dr Boyan Zlatkov was given the opportunity to extract the Tortricoidea. The remaining material was retained and sent by post to England after the end of each year, when any Pyraloidea were extracted, preserved and then named by CWP.

\section{Targetted survey in 2017}

During 2017, CWP undertook self-funded, targetted collecting of Pyraloidea in Serbia on several nights in June in the company of $\mathrm{PJ}$; the sites were pre-selected by $\mathrm{PJ}$, in order to provide a representative cross-section of different habitats to be sampled. A total of 8 nights collecting had been proposed, with the intention of spending half these in the west of Serbia and the other half in the east of the country. Unfortunately, heavy rain reduced this to 7 nights - three in the west and four in the east.

Apart from captures made by a hand-held net, the pyraloid moths were sampled in 2017 using light trapping, as follows:

- Up to four "Robinson-pattern" traps were fitted with a 125 watt mercury-vapour bulb; these traps are in frequent use in Great Britain and are not only effective in attracting moths but are also efficient in retaining captured moths in the absence of anaesthetics. All traps were operated from the dusk until just beyond daybreak. These traps regularly catch very large numbers of moths making examination of the catch difficult; consequently, chloroform (trichloromethane) was introduced as an anaesthetic before opening the trap.

- A separate 125 watt mercury-vapour bulb was suspended between 1.5 and 2 metres above the ground 
in front of a white sheet, itself suspended vertically from the side of CWP's specially adapted Landrover vehicle. Moths were hand-collected from the sheet.

- Up to five "Hungarian" traps were also used. These consist of an 8 watt actinic "black light" tube suspended over a sealed, plastic bucket. The catch in these traps is significantly lower than that in the Robinson-pattern traps; the traps are charged with chloroform at set-up.

At each site, the traps used were positioned at a varying distances from each other - the primary consideration being that they were placed in differing habitat areas. No trap was set in a position from where it was possible to observe directly another light.

The combination of these two sampling techniques produced samples from a total of 40 separate localities on a total of 53 different dates as follows:

- Serbia:

17 sites on 23 dates

- Albania: 11 sites on 16 dates

- Montenegro: 2 sites on 2 dates

- Macedonia: 10 sites on 12 dates

The geographical spread of these sites is indicated in Fig. 1.

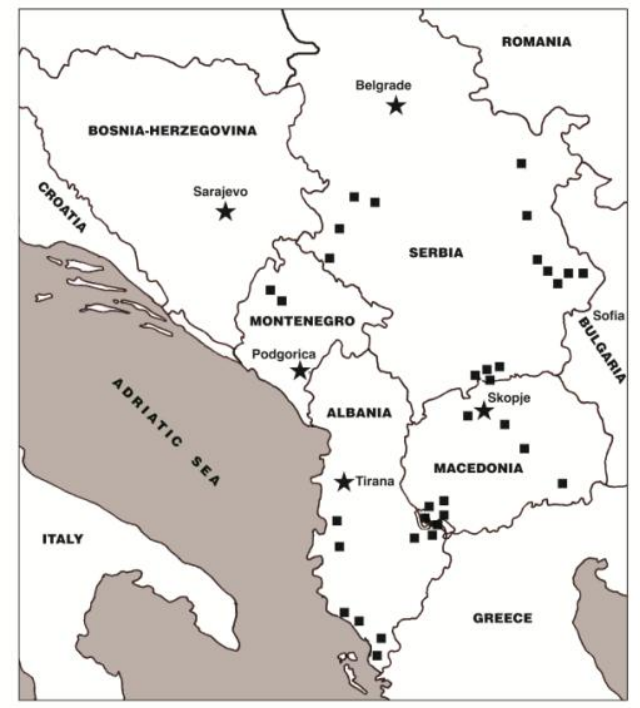

Figure 1. Collecting localities in 2014, 2016 and 2017 are indicated approximately by the solid black squares. Some squares represent more than one locality. The positions of the squares are not precise; refer to Tables 1,2 and 3 for precise coordinates.

Table 1. Details of sampled sites within Serbia (17 sites on 23 dates - "sites 1 - 23"). Serbian place names are based on Jovanović (2010) and those from Albania on Friese (1967).

\begin{tabular}{|c|c|c|c|c|c|}
\hline $\begin{array}{l}\text { Site } \\
\text { Ref }\end{array}$ & Place name & Coordinates & $\begin{array}{l}\text { Altitude } \\
\text { (metres) }\end{array}$ & $\begin{array}{c}\text { Date } \\
\text { sampled }\end{array}$ & Leg. \\
\hline 1 & Jelašnička Klisura & $43^{\circ} 16^{\prime} 36^{\prime \prime} \mathrm{N}: 22^{\circ} 04^{\prime} 09^{\prime \prime} \mathrm{E}$ & 345 & 18.iii.2014 & SB \& AN \\
\hline 3 & Suva Planina, above Bojanine Vode & $43^{\circ} 12^{\prime} 55^{\prime \prime} \mathrm{N}: 22^{\circ} 07: 26^{\prime \prime} \mathrm{E}$ & 1030 & 19.vi.2017 & CWP \& PJ \\
\hline 5 & $\begin{array}{l}\text { Pirot, Mt. Vidlič, above Kamenolom Kitka to Crni Vrh } \\
\text { Summit }\end{array}$ & $43^{\circ} 10^{\prime} 49^{\prime \prime} \mathrm{N}: 22^{\circ} 38^{\prime} 35^{\prime \prime} \mathrm{E}$ & 870 & 4.vii.2014 & SB \& AN \\
\hline 6 & Pirot Region, near Krupac village & $43^{\circ} 06^{\prime} 15^{\prime \prime} \mathrm{N}: 22^{\circ} 42^{\prime} 55^{\prime \prime} \mathrm{E}$ & 688 & 5.vii.2014 & SB \& AN \\
\hline 7 & Zvijezda, Savina Voda, near Jabuka Pass & $43^{\circ} 22^{\prime} 02^{\prime \prime} \mathrm{N}: 19^{\circ} 33^{\prime} 07^{\prime \prime} \mathrm{E}$ & 1117 & 16.vii.2014 & SB \& AN \\
\hline 10 & $\begin{array}{l}\text { Bela Palanka district, Mt. Šljivoviki Vis, above Šljivovik } \\
\text { village }\end{array}$ & $43^{\circ} 08^{\prime} 29^{\prime \prime} \mathrm{N}: 22^{\circ} 23^{\prime} 12^{\prime \prime} \mathrm{E}$ & 925 & $3 . x .2016$ & SB \& AN \\
\hline 11 & Preševo district, above Trnava village & $42^{\circ} 16^{\prime} 18^{\prime \prime} \mathrm{N}: 21^{\circ} 36^{\prime} 47^{\prime \prime} \mathrm{E}$ & 800 & 31.v.2016 & SB \& AN \\
\hline 12 & Preševo district, above Trnava village & $42^{\circ} 16^{\prime} 18^{\prime \prime} \mathrm{N}: 21^{\circ} 36^{\prime} 47^{\prime \prime} \mathrm{E}$ & 800 & 9.vi.2016 & SB \& AN \\
\hline 13 & Preševo district, above Trnava village & $42^{\circ} 16^{\prime} 18^{\prime \prime} \mathrm{N}: 21^{\circ} 36^{\prime} 07^{\prime \prime} \mathrm{E}$ & 800 & 30.vi.2016 & SB \& AN \\
\hline 14 & Preševo district, above Trnava village & $42^{\circ} 16^{\prime} 08^{\prime \prime} \mathrm{N}: 21^{\circ} 36^{\prime} 47^{\prime \prime} \mathrm{E}$ & 800 & 9.vii.2016 & SB \& AN \\
\hline 15 & Svrljiški Timok river gorge, near Niševac village & $43^{\circ} 28^{\prime} 15^{\prime \prime} \mathrm{N}: 22^{\circ} 05^{\prime} 27^{\prime \prime} \mathrm{E}$ & 430 & 4.viii.2016 & SB \& AN \\
\hline
\end{tabular}




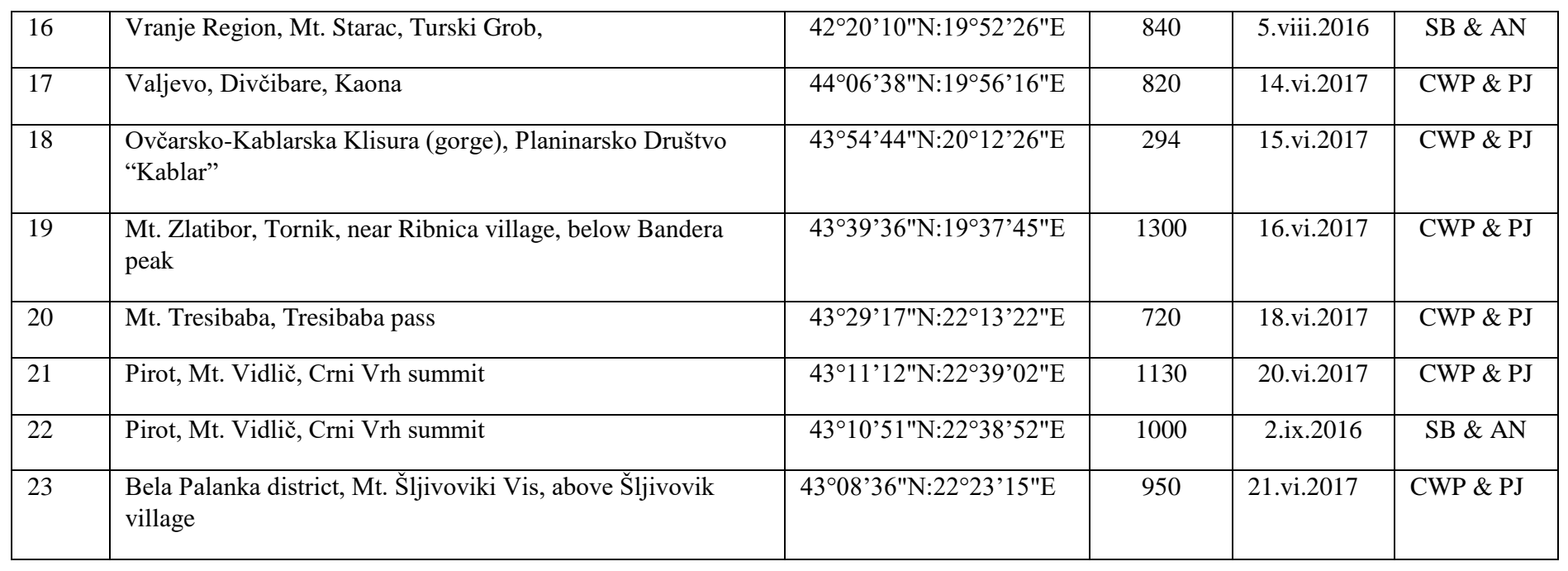

Table 2. Details of sampled sites within Albania (11 sites on 16 dates - "sites 24 - 39").

\begin{tabular}{|c|c|c|c|c|c|}
\hline $\begin{array}{l}\text { Site } \\
\text { Ref }\end{array}$ & Place name & Coordinates & $\begin{array}{l}\text { Altitude } \\
\text { (metres) }\end{array}$ & $\begin{array}{c}\text { Date } \\
\text { sampled }\end{array}$ & Leg. \\
\hline 24 & Korçë region, Devolli Gorge, near Strelçë (Maliq district) & $40^{\circ} 43^{\prime} 18^{\prime \prime N}: 20^{\circ} 31^{\prime} 5 " \mathrm{E}$ & 755 & 5.vi.2016 & SB \& AN \\
\hline 25 & Ionian coast, Palasë, near Dhërmi & $40^{\circ} 10^{\prime} 35^{\prime \prime} \mathrm{N}: 19^{\circ} 36^{\prime} 21^{\prime \prime} \mathrm{E}$ & 274 & 6.vi.2016 & SB \& AN \\
\hline 26 & Delvinë region, Bistricë & $39^{\circ} 55^{\prime} 24^{\prime \prime} \mathrm{N}: 20^{\circ} 07^{\prime} 53^{\prime \prime} \mathrm{E}$ & 116 & 7.vi.2016 & SB \& AN \\
\hline 27 & Delvinë region, Bistricë & $39^{\circ} 55^{\prime} 24^{\prime \prime} \mathrm{N}: 20^{\circ} 07^{\prime} 53^{\prime \prime} \mathrm{E}$ & 116 & 19.x.2016 & SB \& AN \\
\hline 28 & Korçë region, above Kloçë, near the radio mast (= antenna) & $40^{\circ} 41^{\prime} 02^{\prime \prime} \mathrm{N}: 20^{\circ} 41^{\prime} 40^{\prime \prime} \mathrm{E}$ & 1217 & 4.vii. 2016 & SB \& AN \\
\hline 29 & Korçë region, above Drenovë & $40^{\circ} 53^{\prime} 18^{\prime \prime} \mathrm{N}: 20^{\circ} 48^{\prime} 23^{\prime \prime} \mathrm{E}$ & 1050 & 7.vii.2016 & SB \& AN \\
\hline 30 & Korçë region, above Drenovë & $40^{\circ} 53^{\prime} 18^{\prime \prime} \mathrm{N}: 20^{\circ} 48^{\prime} 23 " \mathrm{E}$ & 1050 & 9.viii.2016 & SB \& AN \\
\hline 31 & Korçë region, above Drenovë & $40^{\circ} 53^{\prime} 18^{\prime \prime} \mathrm{N}: 20^{\circ} 48^{\prime} 23^{\prime \prime} \mathrm{E}$ & 1050 & 24.ix.2016 & SB \& AN \\
\hline 32 & Korçë region, above Boboshticë and below Dardhë & $40^{\circ} 32^{\prime} 26^{\prime \prime} \mathrm{N}: 20^{\circ} 47^{\prime} 31^{\prime \prime} \mathrm{E}$ & 1225 & 8.vii.2016 & SB \& AN \\
\hline 33 & $\begin{array}{l}\text { Berat region, Poliçan district, Vukopoles River Gorge } \\
\text { between Ibrollara and Valë }\end{array}$ & $40^{\circ} 33^{\prime} 36^{\prime \prime} \mathrm{N}: 20^{\circ} 05^{\prime} 38^{\prime \prime} \mathrm{E}$ & 217 & 10.viii.2016 & SB \& AN \\
\hline 34 & $\begin{array}{l}\text { Berat region, Poliçan district, Vukopoles River Gorge } \\
\text { between Ibrollara and Valë }\end{array}$ & $40^{\circ} 33^{\prime} 36^{\prime \prime} \mathrm{N}: 20^{\circ} 05^{\prime} 38^{\prime \prime} \mathrm{E}$ & 217 & 25.ix.2016 & SB \& AN \\
\hline 35 & $\begin{array}{l}\text { Berat region, Poliçan district, Vukopoles River Gorge } \\
\text { between Ibrollara and Valë }\end{array}$ & $40^{\circ} 33^{\prime} 36^{\prime \prime} \mathrm{N}: 20^{\circ} 05^{\prime} 38^{\prime \prime} \mathrm{E}$ & 217 & $16 . x .2016$ & SB \& AN \\
\hline 36 & Lushnjë region, above Stan-Karbunarë & $40^{\circ} 55^{\prime} 03^{\prime \prime} \mathrm{N}: 19^{\circ} 43^{\prime} 57^{\prime \prime E}$ & 64 & 11.viii.2016 & SB \& AN \\
\hline 37 & Korçë region, Mt. Ivan, above Zvezdë village & $40^{\circ} 43^{\prime} 59^{\prime \prime N}: 20^{\circ} 52^{\prime} 49^{\prime \prime} \mathrm{E}$ & 1088 & 23.ix.2016 & SB \& AN \\
\hline 38 & $\begin{array}{l}\text { Ionian coast, Dhërmi district, below Ilias at St. Theodor } \\
\text { Monastery }\end{array}$ & $40^{\circ} 07^{\prime} 52^{\prime \prime} \mathrm{N}: 19^{\circ} 39^{\prime} 19^{\prime \prime} \mathrm{E}$ & 153 & 17.x.2016 & SB \& AN \\
\hline 39 & Ionian coast, between Butrint and Sarandë, near Ksamil & $39^{\circ} 48^{\prime} 31^{\prime \prime N}: 20^{\circ} 00^{\prime} 27^{\prime \prime E}$ & 56 & 18.x.2016 & SB \& AN \\
\hline
\end{tabular}


Table 3. Details of sampled sites within Montenegro (2 sites on 2 dates) and Macedonia (10 sites on 12 dates) - sites 40 - 53.

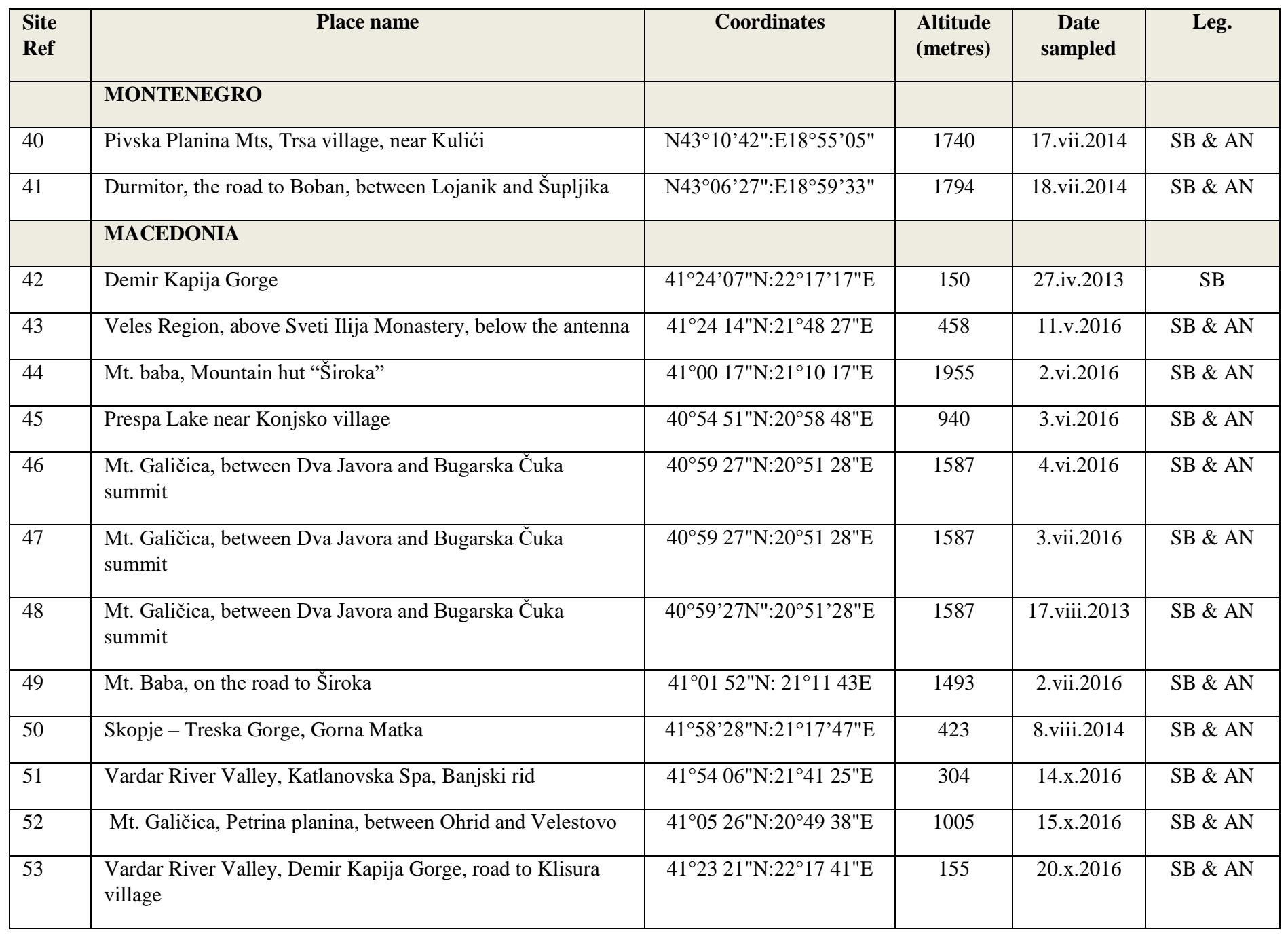

\section{Laboratory aspects}

Regrettably, most of the material in the trap debris retained by $\mathrm{SB}$ and AN was in very poor condition. The specimens that remained dry tended to be lacking in legs, antennae or palpi; other samples had become "greasy" so that external morphological features were obliterated. Consequently, almost without exception moths were named by genitalia examination. Where there was any ambiguity, the "problem" moths were degreased by overnight immersion in ethyl acetate so that characters of the wing patterns might become visible; a small proportion, mostly females, could not be named. The decomposed nature of most material rendered it unsuitable for any eventual molecular analysis and with rather few exceptions, these specimens were discarded.

Material from active sampling by CWP in 2017 tended to be in better condition and in some cases identification could be based on wing morphology alone. Voucher specimens from this expedition are retained in the CWP collection in England.

All identifications, including all genitalia examinations, were undertaken by CWP.

\section{RESULTS}

A grand total of 173 species of Pyraloidea is reported here, comprising 97 species of Crambidae and 76 taxa within the Pyralidae. The number recorded in each political unit is indicated in Table 4.

Table 4. Numbers of pyraloid taxa recorded in each country examined during the present survey.

\begin{tabular}{|l|c|c|c|}
\hline \multirow{2}{*}{ Region } & \multicolumn{3}{|c|}{ Number of } \\
\cline { 2 - 4 } & Crambidae & Pyralidae & all Pyraloidea \\
\hline Serbia & 63 & 57 & 120 \\
\hline Albania & 62 & 49 & 111 \\
\hline Macedonia & 25 & 31 & 56 \\
\hline Montenegro & 11 & 8 & 19 \\
\hline All areas: & $\mathbf{9 7}$ & $\mathbf{7 6}$ & $\mathbf{1 7 3}$ \\
\hline
\end{tabular}


At each of this overall total of 40 sites, the catches from the several different traps used on the same night were pooled to provide a single date sample for that site. These 53 site/date sample points are summarised and allocated an identifying number in Tables $5-7$. Lists for each of the 53 separate points were then collated.
In order to provide a permanent record that will be of value to future researchers into the fauna of this geographical region, the names of all species recorded on each of the 53 dates are presented in Table 5 (for Serbia), Table 6 (for Albania) and Table 7 (for Montenegro and Macedonia).

Table 5. Inventory of recorded species at sites in Serbia.

\begin{tabular}{|c|c|c|c|c|c|c|c|c|c|c|c|c|c|c|c|c|c|c|c|c|c|c|c|}
\hline Site ref $\rightarrow$ & 1 & 2 & 3 & 4 & 5 & 6 & 7 & 8 & 9 & 10 & 11 & 12 & 13 & 14 & 15 & 16 & 17 & 18 & 19 & 20 & 21 & 22 & 23 \\
\hline \multicolumn{24}{|l|}{$\operatorname{tax} 0 n \downarrow$} \\
\hline \multicolumn{24}{|l|}{ Crambidae } \\
\hline \multicolumn{24}{|l|}{ Crambinae } \\
\hline $\begin{array}{l}\text { Chrysoteuchia } \\
\text { culmella (Linnaeus, } \\
1758 \text { ) }\end{array}$ & & & + & & & & & & & & & & + & & & & + & + & & + & & & \\
\hline $\begin{array}{l}\text { Crambus ericella } \\
\text { (Hübner, 1813) }\end{array}$ & & & & & & & & & & & & & & & & & + & & & & & & \\
\hline $\begin{array}{l}\text { Crambus } \\
\text { lathoniellus } \\
\text { (Zincken, 1817) }\end{array}$ & & & & & & & & & & & & & & & & & + & + & + & & + & & \\
\hline $\begin{array}{l}\text { Crambus pascuella } \\
\text { (Linnaeus, 1758) }\end{array}$ & & & & & & & & & & & & & & & & & + & & & & & & \\
\hline $\begin{array}{l}\text { Crambus pratella } \\
\text { (Linnaeus, 1758) }\end{array}$ & & & & & & & & & & & & & & & & & & + & + & & & & \\
\hline $\begin{array}{l}\text { Angustalius } \\
\text { malacellus } \\
\text { (Duponchel, 1836) }\end{array}$ & & & & & & & & & & & & & & & & & + & & & & & & \\
\hline $\begin{array}{l}\text { Agriphila tristella } \\
\text { ([Denis \& } \\
\text { Schiffermüller], } \\
1775 \text { ) }\end{array}$ & & & & & & & & & & & & & & & & & & & & & & + & \\
\hline $\begin{array}{l}\text { Catoptria } \\
\text { confusella } \\
\text { (Staudinger, 1881) }\end{array}$ & & & & & & & & & & & & & & & + & & & & & & & + & \\
\hline $\begin{array}{l}\text { Catoptria falsella } \\
\text { ([Denis \& } \\
\text { Schiffermüller], } \\
1775 \text { ) }\end{array}$ & & & & & + & & + & & & & & & & + & & & & & & & & & + \\
\hline $\begin{array}{l}\text { Catoptria } \\
\text { lythargyrella } \\
\text { (Hübner, 1796) }\end{array}$ & & & & & & & & & & + & & & & & & & & & & & & + & + \\
\hline $\begin{array}{l}\text { Catoptria myella } \\
\text { (Hübner, 1796) }\end{array}$ & & & + & & & & & & & & & & & & & & & & & & & & \\
\hline $\begin{array}{l}\text { Catoptria mytilella } \\
\text { (Hübner, 1805) }\end{array}$ & & & & & & & + & & & & & & & & & & & & & & & & \\
\hline $\begin{array}{l}\text { Catoptria } \\
\text { osthelderi (de } \\
\text { Lattin, 1950) }\end{array}$ & & & & & & & & & & & & & & & & & + & & & & & & \\
\hline $\begin{array}{l}\text { Catoptria pinella } \\
\text { (Linnaeus, 1758) }\end{array}$ & & & & & + & & + & + & & & & & & + & + & & & & & & & & + \\
\hline
\end{tabular}




\begin{tabular}{|c|c|c|c|c|c|c|c|c|c|c|c|c|c|c|c|c|c|c|c|c|c|c|c|}
\hline Site ref $\rightarrow$ & 1 & 2 & 3 & 4 & 5 & 6 & 7 & 8 & 9 & 10 & 11 & 12 & 13 & 14 & 15 & 16 & 17 & 18 & 19 & 20 & 21 & 22 & 23 \\
\hline \multicolumn{24}{|l|}{ taxon $\downarrow$} \\
\hline $\begin{array}{l}\text { Xanthocrambus } \\
\text { saxonellus } \\
\text { (Zincken, 1821) }\end{array}$ & & & & & + & & & + & & & & & + & + & & & & & & & + & & \\
\hline $\begin{array}{l}\text { Chrysocrambus } \\
\text { cassentiellus } \\
\text { (Herrich-Schäffer } \\
{[1848] \text { ) }}\end{array}$ & & & & & & & & & & & & & & & & & + & & & & & & \\
\hline $\begin{array}{l}\text { Chrysocrambus } \\
\text { craterella (Scopoli, } \\
1763 \text { ) }\end{array}$ & & + & & + & + & & + & & & & & + & + & & & & + & + & & + & & & + \\
\hline $\begin{array}{l}\text { Thisanotia } \\
\text { chrysonuchella } \\
\text { (Scopoli, 1763) }\end{array}$ & & & & + & & & + & & + & & & & & & & & + & + & + & & + & & + \\
\hline $\begin{array}{l}\text { Platytes cerussella } \\
\text { ([Denis \& } \\
\text { Schiffermüller], } \\
1775 \text { ) }\end{array}$ & & & & & & & & & & & & & & & & & & & + & + & + & & \\
\hline \multicolumn{24}{|l|}{ Scopariinae } \\
\hline $\begin{array}{l}\text { Scoparia } \\
\text { ambigualis } \\
\text { (Treitschke, 1829) }\end{array}$ & & & & & & & & & & & & & & & & & & & & & & & + \\
\hline $\begin{array}{l}\text { Scoparia } \\
\text { basistrigalis } \\
\text { Knaggs, } 1866\end{array}$ & & & & & & & & & & & & & & + & & & + & + & & & + & & \\
\hline $\begin{array}{l}\text { Scoparia ingratella } \\
\text { (Zeller, 1846) }\end{array}$ & & + & & & & & & & & & & & & & & & + & & + & + & + & & \\
\hline $\begin{array}{l}\text { Scoparia } \\
\text { perplexella (Zeller, } \\
1839)\end{array}$ & & & & & & & + & & & & & & & & & & & & + & & & & \\
\hline $\begin{array}{l}\text { Scoparia pyralella } \\
\text { ([Denis \& } \\
\text { Schiffermüller], } \\
1775 \text { ) }\end{array}$ & & & & & + & & + & & & & & & & & & & + & & + & + & + & & + \\
\hline $\begin{array}{l}\text { Scoparia subfusca } \\
\text { Haworth, } 1811\end{array}$ & & & & & & & & & & & & & & & & & + & & & & & & \\
\hline $\begin{array}{l}\text { Eudonia lacustrata } \\
\text { Panzer, } 1804\end{array}$ & & & & & & & + & & & & & & & & & & & & & & + & & \\
\hline $\begin{array}{l}\text { Eudonia } \\
\text { mercurella } \\
\text { (Linnaeus, 1758) }\end{array}$ & & & & & + & & & & & & & & & & & & & & & & & & \\
\hline $\begin{array}{l}\text { Eudonia sudetica } \\
\text { (Zeller, 1839) }\end{array}$ & & & & & & & + & & & & & & & & + & & & & & & + & & + \\
\hline Cybalomiinae & & & & & & & & & & & & & & & & & & & & & & & \\
\hline $\begin{array}{l}\text { Hyperlais } \\
\text { dulcinalis } \\
\text { (Treitschke, 1835) }\end{array}$ & & & & & & & + & & & & & + & + & & & & + & & & & & & \\
\hline
\end{tabular}




\begin{tabular}{|c|c|c|c|c|c|c|c|c|c|c|c|c|c|c|c|c|c|c|c|c|c|c|c|}
\hline Site ref $\rightarrow$ & 1 & 2 & 3 & 4 & 5 & 6 & 7 & 8 & 9 & 10 & 11 & 12 & 13 & 14 & 15 & 16 & 17 & 18 & 19 & 20 & 21 & 22 & 23 \\
\hline $\operatorname{tax} 0 n \downarrow$ & & & & & & & & & & & & & & & & & & & & & & & \\
\hline Odontiinae & & & & & & & & & & & & & & & & & & & & & & & \\
\hline $\begin{array}{l}\text { Cynaeda dentalis } \\
\text { ([Denis \& } \\
\text { Schiffermüller], } \\
1775)\end{array}$ & & & & & & & & + & & & & & & & & & & & & & & & \\
\hline $\begin{array}{l}\text { Cynaeda pustulalis } \\
\text { (Hübner, 1823) }\end{array}$ & & & & & + & & & & & & & & & & & & & & & & + & & + \\
\hline $\begin{array}{l}\text { Ephelis cruentalis } \\
\text { (Geyer, 1832) }\end{array}$ & & & & & & & & + & & & & & + & & & & & & & & & & \\
\hline $\begin{array}{l}\text { Eurrhypis } \\
\text { pollinalis ([Denis } \\
\text { \& Schiffermüller], } \\
1775 \text { ) }\end{array}$ & & & & & & & & & & & & & & & & & & & & & & & + \\
\hline Evergestinae & & & & & & & & & & & & & & & & & & & & & & & \\
\hline $\begin{array}{l}\text { Evergestis aenealis } \\
\text { ([Denis \& } \\
\text { Schiffermüller], } \\
1775 \text { ) }\end{array}$ & & & & + & & & & & + & & + & & & & & & + & + & + & & & & + \\
\hline $\begin{array}{l}\text { Evergestis limbata } \\
\text { (Linnaeus, 1767) }\end{array}$ & & & & & & & & & & & & & & & & & & & & & & & + \\
\hline $\begin{array}{l}\text { Evergestis } \\
\text { mundalis (Guenée, } \\
1854)\end{array}$ & & & & & & & & & & & & & & + & & & & & & & & & \\
\hline $\begin{array}{l}\text { Evergestis } \\
\text { sophialis } \\
\text { (Fabricius, 1787) }\end{array}$ & & & & & + & & & & & & & & & & & & & & & + & + & & + \\
\hline Pyraustinae & & & & & & & & & & & & & & & & & & & & & & & \\
\hline $\begin{array}{l}\text { Loxostege } \\
\text { aeruginalis } \\
\text { (Hübner, 1796) }\end{array}$ & & & & + & + & & & & + & & & + & & & & & & & & & & & + \\
\hline $\begin{array}{l}\text { Paratalanta } \\
\text { pandalis (Hübner, } \\
1825)\end{array}$ & & & & & & & & & & & & & & & & + & & & & & & & + \\
\hline $\begin{array}{l}\text { Pyrausta aurata } \\
\text { (Scopoli, 1763) }\end{array}$ & & & & & + & & & + & & & & & & + & & & & & & & + & & + \\
\hline $\begin{array}{l}\text { Pyrausta cingulata } \\
\text { (Linnaeus, 1758) }\end{array}$ & & & & & & & & & & & & + & & & & & & & & & & & \\
\hline $\begin{array}{l}\text { Pyrausta despicata } \\
\text { (Scopoli, 1763) }\end{array}$ & & & & & & & & & & + & + & & & & & & + & & & & & & + \\
\hline $\begin{array}{l}\text { Pyrausta nigrata } \\
\text { (Scopoli, 1763) }\end{array}$ & & & & & & & & & & & & & & & & + & & & & & & & \\
\hline $\begin{array}{l}\text { Pyrausta } \\
\text { purpuralis } \\
\text { (Linnaeus, 1758) }\end{array}$ & & & & & & & + & & & + & + & & + & & & + & & & & & & & \\
\hline $\begin{array}{l}\text { Pyrausta } \\
\text { sanguinalis } \\
\text { (Linnaeus, 1767) }\end{array}$ & & & & & + & & & & & + & + & & & & + & & & & & & & & + \\
\hline
\end{tabular}




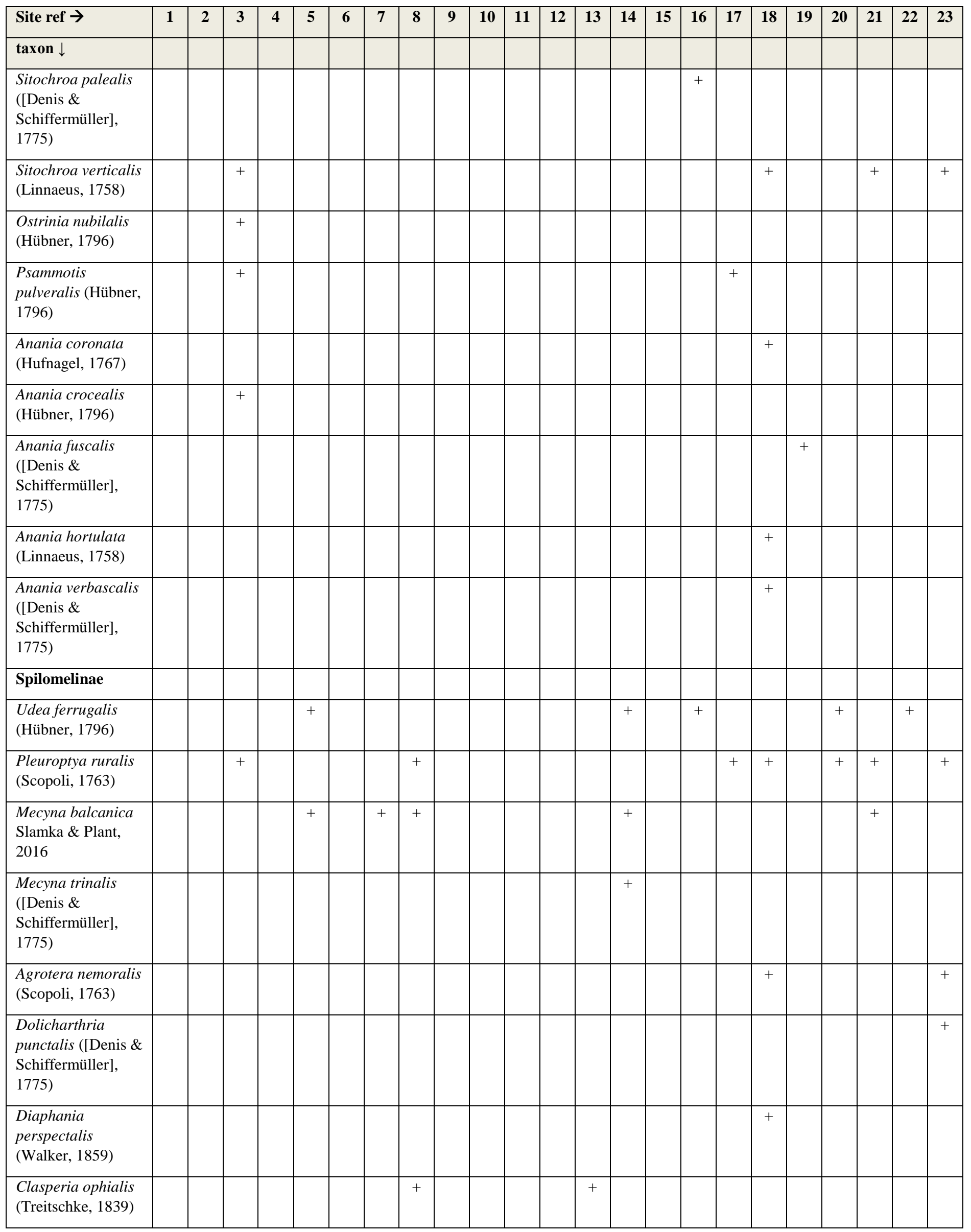




\begin{tabular}{|c|c|c|c|c|c|c|c|c|c|c|c|c|c|c|c|c|c|c|c|c|c|c|c|}
\hline Site ref $\rightarrow$ & 1 & 2 & 3 & 4 & 5 & 6 & 7 & 8 & 9 & 10 & 11 & 12 & 13 & 14 & 15 & 16 & 17 & 18 & 19 & 20 & 21 & 22 & 23 \\
\hline $\operatorname{tax} 0 n \downarrow$ & & & & & & & & & & & & & & & & & & & & & & & \\
\hline $\begin{array}{l}\text { Nomophila } \\
\text { noctuella ([Denis } \\
\text { \& Schiffermüller], } \\
1775)\end{array}$ & & & & & & & & & & & & & & & & + & & & & & & + & + \\
\hline Pyralidae & & & & & & & & & & & & & & & & & & & & & & & \\
\hline Phycitinae & & & & & & & & & & & & & & & & & & & & & & & \\
\hline $\begin{array}{l}\text { Bradyrrhoa } \\
\text { gilveolella } \\
\text { (Treitschke, 1832) }\end{array}$ & & & & & & & & & & & & & + & + & & & & & & & & & \\
\hline $\begin{array}{l}\text { Oncocera } \\
\text { semirubella } \\
(\text { Scopoli, 1763) }\end{array}$ & & & & & & & + & & & & & + & & + & & + & + & + & & & & & \\
\hline $\begin{array}{l}\text { Laodamia faecella } \\
\text { (Zeller, 1839) }\end{array}$ & & & & & + & + & + & & & & & & & & & & & & & & & & \\
\hline $\begin{array}{l}\text { Rhodophaea } \\
\text { formosa (Haworth, } \\
1811 \text { ) }\end{array}$ & & & & & & & & & & & & & & & & & + & & & & & & \\
\hline $\begin{array}{l}\text { Sciota fumella } \\
\text { (Eversmann, 1844) }\end{array}$ & & & + & & & & & & & & & & & & & & + & + & & & & & \\
\hline $\begin{array}{l}\text { Sciota hostilis } \\
\text { (Stephens, 1834) }\end{array}$ & & + & & & & & & & & & & & & & & & & & & & & & \\
\hline $\begin{array}{l}\text { Denticera divisella } \\
\text { (Duponchel, 1842) }\end{array}$ & & & & & + & & & & & & & & & & & & & & & & & & \\
\hline $\begin{array}{l}\text { Pempelia } \\
\text { palumbella ([Denis } \\
\text { \& Schiffermüller], } \\
1775)\end{array}$ & & & + & + & + & & + & & + & + & + & & + & & + & & + & & & & & + & + \\
\hline $\begin{array}{l}\text { Selagia spadicella } \\
\text { (Hübner, 1796) }\end{array}$ & & & & & & & & + & & & & & & + & + & + & & & & & & & \\
\hline $\begin{array}{l}\text { Phycita coronatella } \\
\text { (Guenée, 1845) }\end{array}$ & & & & & & + & & & & & & & & & & & & & & & & + & \\
\hline $\begin{array}{l}\text { Phycita cryptica } \\
\text { Plant \& Slamka, } \\
2016\end{array}$ & & & & & & & & & & & & & & & & & & & & & & + & \\
\hline $\begin{array}{l}\text { Phycita meliella } \\
\text { (Mann, 1864) }\end{array}$ & & & & & & & & + & & & & & + & & + & + & & & & & & & \\
\hline $\begin{array}{l}\text { Phycita poteriella } \\
\text { (Zeller, 1846) }\end{array}$ & & & & & & & & & & & & & & + & & & & & & & & & \\
\hline $\begin{array}{l}\text { Phycita roborella } \\
\text { ([Denis \& } \\
\text { Schiffermüller], } \\
1775)\end{array}$ & & & & & & & + & + & & & & + & & + & + & + & & & & & & & + \\
\hline $\begin{array}{l}\text { Phycita torrenti } \\
\text { Agenjo, } 1963\end{array}$ & & & & & & & & & & & & & + & & & & & & & & & & \\
\hline $\begin{array}{l}\text { Dioryctria } \\
\text { simplicella } \\
\text { Heinemann, } 1863\end{array}$ & & & & & & & & & & & & & & & & & + & & + & & & & \\
\hline
\end{tabular}




\begin{tabular}{|c|c|c|c|c|c|c|c|c|c|c|c|c|c|c|c|c|c|c|c|c|c|c|c|}
\hline Site ref $\rightarrow$ & 1 & 2 & 3 & 4 & 5 & 6 & 7 & 8 & 9 & 10 & 11 & 12 & 13 & 14 & 15 & 16 & 17 & 18 & 19 & 20 & 21 & 22 & 23 \\
\hline $\operatorname{taxon} \downarrow$ & & & & & & & & & & & & & & & & & & & & & & & \\
\hline $\begin{array}{l}\text { Etiella zinckenella } \\
\text { (Treitschke, 1832) }\end{array}$ & & & & & & & & + & & & + & & & + & & + & & & & & & & \\
\hline $\begin{array}{l}\text { Epischnia } \\
\text { prodromella } \\
\text { (Hübner, 1799) }\end{array}$ & & & & & & & & & & & + & & & & + & + & & & + & & & & \\
\hline $\begin{array}{l}\text { Catastia marginea } \\
\text { ([Denis \& } \\
\text { Schiffermüller], } \\
1775)\end{array}$ & & & & & & & & & & & & & & & & & & & & & + & & \\
\hline $\begin{array}{l}\text { Elegia similella } \\
\text { (Zincken, 1818) }\end{array}$ & & + & & & + & & & & & & & & & + & & & + & & + & & + & & \\
\hline $\begin{array}{l}\text { Khorassania } \\
\text { compositella } \\
\text { (Treitschke, 1835) }\end{array}$ & + & & & & & & & & & & & & & & & & & & & & & & \\
\hline $\begin{array}{l}\text { Trachonitis } \\
\text { cristella (Hübner, } \\
1796 \text { ) }\end{array}$ & & & & & & & & & & & & & & & & & & + & & & & & \\
\hline $\begin{array}{l}\text { Delplanqueia } \\
\text { dilutella ([Denis \& } \\
\text { Schiffermüller], } \\
\text { 1775) s. str. }\end{array}$ & & & & & + & & & & & & + & + & + & + & & & & & + & & + & & + \\
\hline $\begin{array}{l}\text { Delplanqueia } \\
\text { inscriptella } \\
\text { (Duponchel, 1836) }\end{array}$ & & & & & & & + & & & & + & & & & + & & & & & & & & \\
\hline $\begin{array}{l}\text { Pempeliella } \\
\text { bulgarica Slamka } \\
\& \text { Plant, } 2016\end{array}$ & & & & & & & & + & & & & & & & & & & & & & & & \\
\hline $\begin{array}{l}\text { Pempeliella } \\
\text { ornatella ([Denis \& } \\
\text { Schiffermüller], } \\
1775)\end{array}$ & & & + & + & + & + & + & & + & & + & + & & & & & & & + & + & + & & \\
\hline $\begin{array}{l}\text { Pempeliella } \\
\text { sororiella Zeller, } \\
1839\end{array}$ & & & & & + & & & & & & & & & & & & & & & & + & & \\
\hline $\begin{array}{l}\text { Hypochalcia } \\
\text { ahenella ([Denis \& } \\
\text { Schiffermüller], } \\
1775)\end{array}$ & & & + & & & & + & & & & & & & & & & & & & + & + & & + \\
\hline $\begin{array}{l}\text { Acrobasis } \\
\text { advenella } \\
\text { (Zincken, 1818) }\end{array}$ & & & & & + & & & & & & & & & & & & & & & & & & \\
\hline $\begin{array}{l}\text { Acrobasis } \\
\text { consociella } \\
\text { (Hübner, 1813) }\end{array}$ & & & & & & & & & & & & & + & + & & & & & & & + & & \\
\hline $\begin{array}{l}\text { Acrobasis } \\
\text { fallouella } \\
\text { (Ragonot, 1871) }\end{array}$ & & & & & & & & + & & & & + & & + & & & & & & & & & \\
\hline $\begin{array}{l}\text { Acrobasis legatea } \\
\text { (Haworth, 1811) }\end{array}$ & & & & & & & & & & & & & & & & & & & & & & & + \\
\hline
\end{tabular}




\begin{tabular}{|c|c|c|c|c|c|c|c|c|c|c|c|c|c|c|c|c|c|c|c|c|c|c|c|}
\hline Site ref $\rightarrow$ & 1 & 2 & 3 & 4 & 5 & 6 & 7 & 8 & 9 & 10 & 11 & 12 & 13 & 14 & 15 & 16 & 17 & 18 & 19 & 20 & 21 & 22 & 23 \\
\hline taxon $\downarrow$ & & & & & & & & & & & & & & & & & & & & & & & \\
\hline $\begin{array}{l}\text { Acrobasis } \\
\text { marmorea } \\
\text { (Haworth, 1811) }\end{array}$ & & & + & & & & & & & & & & & & & & & & & + & + & & + \\
\hline $\begin{array}{l}\text { Acrobasis sodalella } \\
\text { (Zeller, 1858) }\end{array}$ & & & & & & & & & & & & + & & + & & & & & & & & & \\
\hline $\begin{array}{l}\text { Acrobasis suavella } \\
\text { (Zincken, 1818) }\end{array}$ & & & & & & & & & & & & & & & & & & & & & & & + \\
\hline $\begin{array}{l}\text { Acrobasis } \\
\text { tumidana ([Denis } \\
\& \text { Schiffermüller], } \\
1775)\end{array}$ & & & & & & & + & & & & & & & + & & + & & & & & & & \\
\hline $\begin{array}{l}\text { Episcythrastis } \\
\text { tabidella (Mann, } \\
1864)\end{array}$ & & & & & & & & + & & & & & & & & + & & & & & & & \\
\hline $\begin{array}{l}\text { Eurhodope } \\
\text { cirrigerella } \\
\text { (Zincken, 1818) }\end{array}$ & & & & + & & & & & & & & & & & & & & & & & & & \\
\hline $\begin{array}{l}\text { Myelois } \\
\text { circumvoluta } \\
\text { (Fourcroy, 1785) }\end{array}$ & & & & & & & & + & & & & & + & + & & & & & & & & & + \\
\hline $\begin{array}{l}\text { Euzopherodes } \\
\text { charlottae (Rebel, } \\
\text { 1914) }\end{array}$ & & & & & & & & & & & & & & & & + & & & & & & & \\
\hline $\begin{array}{l}\text { Ancylosis } \\
\text { cinnamomella } \\
\text { (Duponchel, 1836) }\end{array}$ & & & & & + & + & & + & & & + & + & + & + & + & & & & & & + & & + \\
\hline $\begin{array}{l}\text { Homoeosoma } \\
\text { nebulella ([Denis } \\
\text { \& Schiffermüller], } \\
1775)\end{array}$ & & + & & & & & & & & & & & & & + & + & & & & & & & \\
\hline $\begin{array}{l}\text { Homoeosoma } \\
\text { sinuella (Fabricius, } \\
1794 \text { ) }\end{array}$ & & & & & & & & & & & & & & & & + & + & & & + & & & \\
\hline $\begin{array}{l}\text { Phycitodes } \\
\text { bentickella Pierce, } \\
1937\end{array}$ & & & & & & & + & & & & & & & & & & & & & & & & \\
\hline $\begin{array}{l}\text { Ephestia kuehniella } \\
\text { (Zeller, 1879) }\end{array}$ & & & & & & & & & & & & & & + & & & & & & & & & \\
\hline $\begin{array}{l}\text { Ephestia woodiella } \\
\text { Richards \& } \\
\text { Thompson, } 1932\end{array}$ & & & & & & & & + & & & & & & & & & & & & & & & \\
\hline $\begin{array}{l}\text { Cadra furcatella } \\
\text { (Herrich-Schäffer, } \\
1849 \text { ) }\end{array}$ & & + & & & & & & & & & & & & & & & & & & & & & \\
\hline $\begin{array}{l}\text { Ematheudes } \\
\text { punctella } \\
\text { (Treitschke, 1833) }\end{array}$ & & & & & & & & + & & & & & & & & & & & & & & & \\
\hline
\end{tabular}




\begin{tabular}{|c|c|c|c|c|c|c|c|c|c|c|c|c|c|c|c|c|c|c|c|c|c|c|c|}
\hline Site ref $\rightarrow$ & 1 & 2 & 3 & 4 & 5 & 6 & 7 & 8 & 9 & 10 & 11 & 12 & 13 & 14 & 15 & 16 & 17 & 18 & 19 & 20 & 21 & 22 & 23 \\
\hline \multicolumn{24}{|l|}{$\operatorname{taxon} \downarrow$} \\
\hline \multicolumn{24}{|l|}{ Pyralinae } \\
\hline $\begin{array}{l}\text { Endotricha } \\
\text { flammealis ([Denis } \\
\& \text { Schiffermüller], } \\
1775 \text { ) }\end{array}$ & & + & & & & & + & + & & & & & & + & + & + & & & & & & & \\
\hline $\begin{array}{l}\text { Synaphe punctalis } \\
\text { (Fabricius, 1775) }\end{array}$ & & & & & & & & & & & & & & & & + & & & & & + & & \\
\hline $\begin{array}{l}\text { Pyralis farinalis } \\
\text { (Linnaeus, 1758) }\end{array}$ & & & + & & & & & & & & & & & & & & & & & & & & + \\
\hline $\begin{array}{l}\text { Pyralis regalis } \\
\text { ([Denis \& } \\
\text { Schiffermüller], } \\
1775 \text { ) }\end{array}$ & & + & & & + & & & + & & & & & & & & & & & & & & & + \\
\hline $\begin{array}{l}\text { Stemmatophora } \\
\text { brunnealis } \\
\text { (Treitschke, 1839) }\end{array}$ & & & & & & & & & & & & & & & & + & & & & & & + & + \\
\hline $\begin{array}{l}\text { Stemmatophora } \\
\text { honestalis } \\
\text { (Treitschke, 1829) }\end{array}$ & & & & & + & & & & & & & & & & & & & & & & & & \\
\hline $\begin{array}{l}\text { Hypsopygia } \\
\text { costalis (Fabricius, } \\
1775 \text { ) }\end{array}$ & & + & & & & & & & & & & & & & & & + & + & & & + & & \\
\hline $\begin{array}{l}\text { Hypsopygia } \\
\text { glaucinalis } \\
\text { (Linnaeus, 1758) }\end{array}$ & & + & & & & & & & & & & & & & & + & & & & & + & & \\
\hline $\begin{array}{l}\text { Hypsopygia } \\
\text { rubidalis ([Denis \& } \\
\text { Schiffermüller], } \\
1775)\end{array}$ & & & & & + & & & + & & & & & & & & + & + & & & & & & \\
\hline $\begin{array}{l}\text { Totals per } \\
\text { site/date }\end{array}$ & 1 & 10 & 13 & 7 & 25 & 4 & 22 & 22 & 5 & 5 & 11 & 11 & 15 & 25 & 13 & 22 & 27 & 18 & 14 & 12 & 27 & 9 & 35 \\
\hline
\end{tabular}

Table 6. Inventory of recorded species at sites in Albania (sites 24 to 39)

\begin{tabular}{|l|l|l|l|l|l|l|l|l|l|l|l|l|l|l|l|l|}
\hline Site ref $\rightarrow$ & $\mathbf{2 4}$ & $\mathbf{2 5}$ & $\mathbf{2 6}$ & $\mathbf{2 7}$ & $\mathbf{2 8}$ & $\mathbf{2 9}$ & $\mathbf{3 0}$ & $\mathbf{3 1}$ & $\mathbf{3 2}$ & $\mathbf{3 3}$ & $\mathbf{3 4}$ & $\mathbf{3 5}$ & $\mathbf{3 6}$ & $\mathbf{3 7}$ & $\mathbf{3 8}$ & $\mathbf{3 9}$ \\
\hline taxon $\downarrow$ & & & & & & & & & & & & & & & \\
\hline Crambidae & & & & & & & & & & & & & & & & \\
\hline Crambinae & & & & & & & & & & & & & & & & \\
\hline Euchromius bellus (Hübner, 1796) & & & & & & + & & & & & & & & & & \\
\hline Chrysoteuchia culmella (Linnaeus, 1758) & + & & & & & & & & & & & & & & & \\
\hline Crambus pascuella (Linnaeus, 1758) & & & & & & & & & & & + & & & & \\
\hline Angustalius malacellus (Duponchel, 1836) & & & & & & & & & & & & & & + \\
\hline Agriphila brioniella (Zerny, 1914) & & & & & & & & & & & + & & \\
\hline Agriphila tolli (Bleszyński, 1952) & & & & & + & & & & & & & & & + \\
\hline Catoptria falsella ([Denis \& Schiffermüller], 1775) & & & & & & & \\
\hline
\end{tabular}




\begin{tabular}{|c|c|c|c|c|c|c|c|c|c|c|c|c|c|c|c|c|}
\hline Site ref $\rightarrow$ & 24 & 25 & 26 & 27 & 28 & 29 & 30 & 31 & 32 & 33 & 34 & 35 & 36 & 37 & 38 & 39 \\
\hline \multicolumn{17}{|l|}{ taxon $\downarrow$} \\
\hline Catoptria mytilella (Hübner, 1805) & & & & & & & & + & & & & & & & & \\
\hline Xanthocrambus saxonellus (Zincken, 1821) & & & & & & + & & & & & & & & & & \\
\hline Ancylolomia palpella ([Denis \& Schiffermüller], 1775) & & & & & & & & & & & & & & + & & \\
\hline \multicolumn{17}{|l|}{ Scopariinae } \\
\hline Scoparia basistrigalis Knaggs, 1866 & + & & + & & & & & & & & & & & & & \\
\hline Scoparia ingratella (Zeller, 1846) & & & & & & + & & & & & & & & & & \\
\hline Scoparia manifestella (Herrich-Schäffer, 1848) & & & & & & + & & & & & & & & & & \\
\hline Eudonia sudetica (Zeller, 1839) & + & & & & & + & & & & & & & & & & \\
\hline \multicolumn{17}{|l|}{ Cybalomiinae } \\
\hline Hyperlais dulcinalis (Treitschke, 1835) & & & + & & & + & & & & & & & & & & \\
\hline \multicolumn{17}{|l|}{ Acentropinae } \\
\hline Cataclysta lemnata (Linnaeus, 1758) & & & & + & & & & & & & & & & & & \\
\hline Elophila nymphaeata (Linnaeus, 1758) & & & & + & & & & & & & & & & & & \\
\hline \multicolumn{17}{|l|}{ Odontiinae } \\
\hline Hellula undalis (Fabricius, 1781) & & & & & & & & & & & + & & & & & \\
\hline Hydriris ornatalis (Duponchel, 1832) & & & & & & & & & & & + & & & & & \\
\hline \multicolumn{17}{|l|}{ Evergestinae } \\
\hline Evergestis aenealis ([Denis \& Schiffermüller], 1775) & + & & & & & & & & + & & & & & & & \\
\hline Evergestis mundalis (Guenée, 1854) & & & & & & + & & & & & & & & & & \\
\hline Evergestis politalis ([Denis \& Schiffermüller], 1775) & & & + & & & & & & & & & & & & & \\
\hline Evergestis serratalis (Staudinger, 1870) & & & & & & & & + & & & & & & + & & \\
\hline \multicolumn{17}{|l|}{ Pyraustinae } \\
\hline Loxostege aeruginalis (Hübner, 1796) & & & & & + & + & & & & & & & & & & \\
\hline Ecpyrrhorrhoe diffusalis Guenée, 1854 & & & & & & & + & & & & & & & & & \\
\hline Ecpyrrhorrhoe rubiginalis (Hübner, 1796) & & & & & & + & & & & & & & & & & \\
\hline Achyra nudalis (Hübner, 1796) & & & + & & & & & & & & & & + & & & \\
\hline Pyrausta aurata (Scopoli, 1763) & & + & + & + & + & & & & & & + & & + & & & + \\
\hline Pyrausta cingulata (Linnaeus, 1758) & & & & & + & & & & & & & & & & & \\
\hline
\end{tabular}




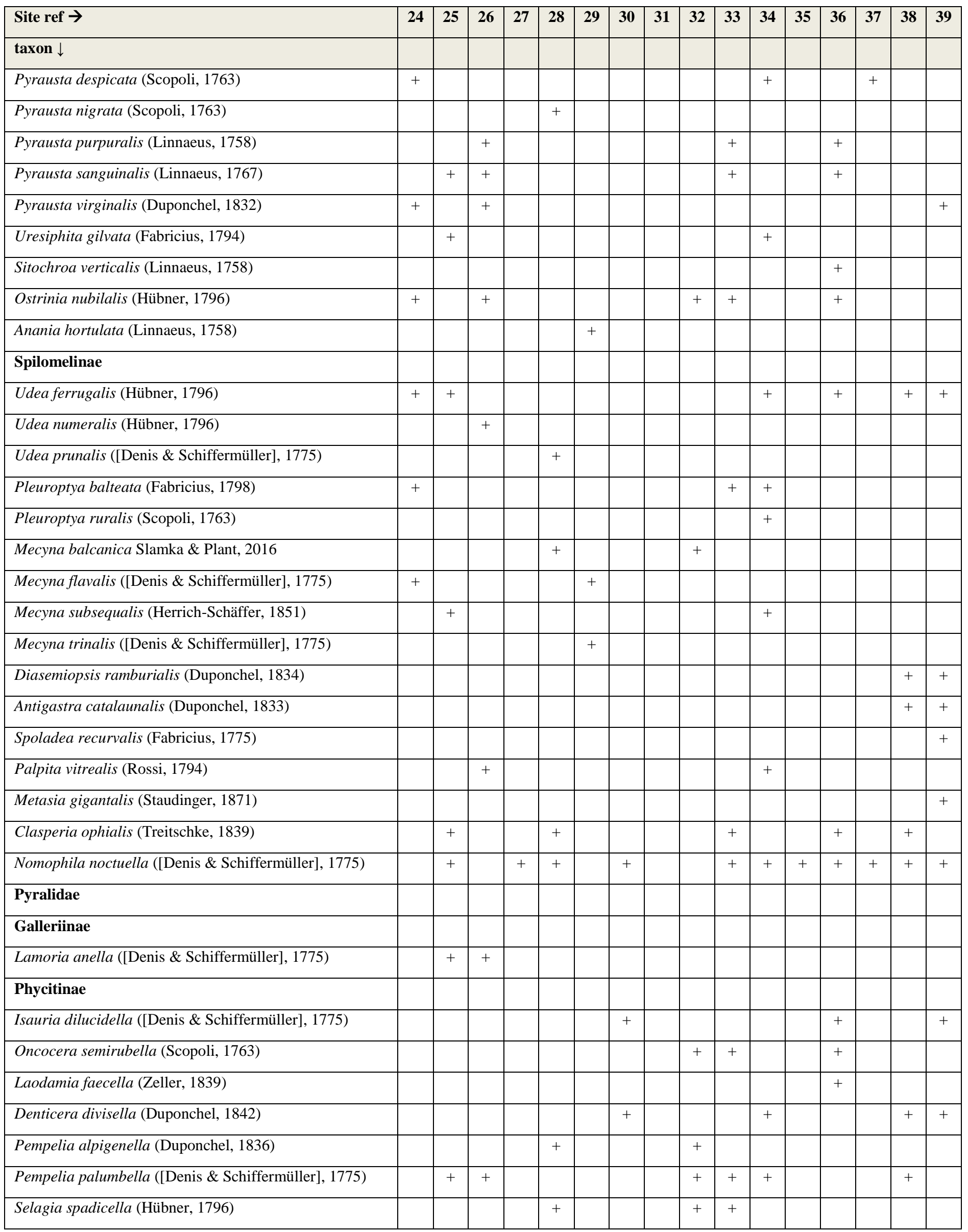




\begin{tabular}{|c|c|c|c|c|c|c|c|c|c|c|c|c|c|c|c|c|}
\hline Site ref $\rightarrow$ & 24 & 25 & 26 & 27 & 28 & 29 & 30 & 31 & 32 & 33 & 34 & 35 & 36 & 37 & 38 & 39 \\
\hline \multicolumn{17}{|l|}{ taxon $\downarrow$} \\
\hline Phycita coronatella (Guenée, 1845) & & & & & + & & & & & & & & & & & \\
\hline Phycita roborella ([Denis \& Schiffermüller], 1775) & & & & & + & & & & & & & & & & & \\
\hline Etiella zinckenella (Treitschke, 1832) & + & + & & & & & & & & & & & + & & + & \\
\hline Epischnia prodromella (Hübner, 1799) & & + & & & & & & & & & & & & & & \\
\hline Elegia fallax (Staudinger, 1881) & + & & & & & & & & & & & & & & & \\
\hline Elegia similella (Zincken, 1818) & + & & & & & & & & & & & & & & & \\
\hline Matilella fusca (Haworth, 1811) & & & & & & & & & & & + & & & & & \\
\hline $\begin{array}{l}\text { Delplanqueia dilutella ([Denis \& Schiffermüller], 1775) } \\
\text { s. str. }\end{array}$ & + & + & + & & & & + & & & + & + & & & & + & \\
\hline Pempeliella bulgarica Slamka \& Plant, 2016 & & & & & & & & & & & & & + & & & \\
\hline Pempeliella ornatella ([Denis \& Schiffermüller], 1775) & + & & & & & & & & + & & & & & & & \\
\hline Pempeliella sororiella Zeller, 1839 & + & & + & & & + & & & & & & & & & & \\
\hline Oxybia transversella (Duponchel, 1836) & & + & + & + & & & & & & + & + & & & & + & \\
\hline Psorosa nucleolella (Möschler, 1866) & & & & & & & & & & & & & & & & + \\
\hline Acrobasis sodalella (Zeller, 1858) & & & & + & & & & & & & & & & & & \\
\hline Eurhodope rosella (Scopoli, 1793) & & + & + & & + & & & & + & & & & & & & \\
\hline Myelois circumvoluta (Fourcroy, 1785) & & & & & + & & & & + & & & & & & & \\
\hline Ancylosis cinnamomella (Duponchel, 1836) & + & & & & & & + & & & & & & & & & \\
\hline Phycitodes binaevella (Hübner, 1813) & & & & & & & & & + & & & & + & & & \\
\hline Phycitodes inquinatella (Ragonot, 1887) & & & + & & & & & & & & & & + & & & \\
\hline Ephestia welseriella (Zeller, 1848) & & & & & & & + & & & & & & & & & \\
\hline Ephestia woodiella Richards \& Thompson, 1932 & & & + & & & & & & & & & & & & + & \\
\hline Cadra furcatella (Herrich-Schäffer, 1849) & + & & & & & & & & & & & & & & & \\
\hline Ematheudes punctella (Treitschke, 1833) & & + & & & & + & & & & & & & + & & & \\
\hline \multicolumn{17}{|l|}{ Pyralinae } \\
\hline Endotricha flammealis ([Denis \& Schiffermüller], 1775) & & + & + & & + & & & & & + & & & & & + & \\
\hline Pyralis farinalis (Linnaeus, 1758) & & & & & & & & & & & & + & & & & \\
\hline Pyralis regalis ([Denis \& Schiffermüller], 1775) & & + & + & & + & + & & & + & & + & & & & + & \\
\hline
\end{tabular}




\begin{tabular}{|l|c|c|c|c|c|c|c|c|c|c|c|c|c|c|c|c|}
\hline Site ref $\rightarrow$ & $\mathbf{2 4}$ & $\mathbf{2 5}$ & $\mathbf{2 6}$ & $\mathbf{2 7}$ & $\mathbf{2 8}$ & $\mathbf{2 9}$ & $\mathbf{3 0}$ & $\mathbf{3 1}$ & $\mathbf{3 2}$ & $\mathbf{3 3}$ & $\mathbf{3 4}$ & $\mathbf{3 5}$ & $\mathbf{3 6}$ & $\mathbf{3 7}$ & $\mathbf{3 8}$ & $\mathbf{3 9}$ \\
\hline taxon $\downarrow$ & & & & & & & & & & & & & & & & \\
\hline Stemmatophora brunnealis (Treitschke, 1839) & & & & & & & & & & & & & + & & & \\
\hline $\begin{array}{l}\text { Stemmatophora combustalis (Fischer Von Röslerstamm, } \\
\text { 1842) }\end{array}$ & & & & + & + & & & & & & & & & & \\
\hline Hypsopygia costalis (Fabricius, 1775) & + & & & + & & & & & & + & & + & + & & & \\
\hline Hypsopygia glaucinalis (Linnaeus, 1758) & + & & & & & & & & & + & + & + & & & & \\
\hline Hypsopygia rubidalis ([Denis \& Schiffermüller], 1775) & & & + & & & & & & & & & & & & & \\
\hline total for each site/date & $\mathbf{2 3}$ & $\mathbf{2 2}$ & $\mathbf{2 5}$ & $\mathbf{7}$ & $\mathbf{2 1}$ & $\mathbf{1 9}$ & $\mathbf{8}$ & $\mathbf{2}$ & $\mathbf{1 6}$ & $\mathbf{1 6}$ & $\mathbf{2 3}$ & $\mathbf{5}$ & $\mathbf{2 2}$ & $\mathbf{6}$ & $\mathbf{1 4}$ & $\mathbf{1 1}$ \\
\hline
\end{tabular}

Table 7. Inventory of recorded species at sites in Montenegro (40 \& 41) and Macedonia (42 - 53).

\begin{tabular}{|c|c|c|c|c|c|c|c|c|c|c|c|c|c|c|}
\hline Site ref $\rightarrow$ & 40 & 41 & 42 & 43 & 44 & 45 & 46 & 47 & 48 & 49 & 50 & 51 & 52 & 53 \\
\hline \multicolumn{15}{|l|}{ taxon $\downarrow$} \\
\hline \multicolumn{15}{|l|}{ Crambidae } \\
\hline \multicolumn{15}{|l|}{ Crambinae } \\
\hline Euchromius bellus (Hübner, 1796) & + & & & & & & & & & & & & & \\
\hline Crambus lathoniellus (Zincken, 1817) & & + & & & & & & & & & & & & \\
\hline Catoptria confusella (Staudinger, 1881) & & & & & & & & & & & + & & & \\
\hline Catoptria domaviella (Rebel, 1904) & & + & & & & & & & & & & & & \\
\hline Catoptria falsella ([Denis \& Schiffermüller], 1775) & & & & & & & & & & & & & & + \\
\hline Scoparia ingratella (Zeller, 1846) & & & & & & & & & & + & & & & \\
\hline Scoparia subfusca Haworth, 1811 & & + & & & & & & & & & & & & \\
\hline Eudonia mercurella (Linnaeus, 1758) & + & & & & & & & & + & & + & & & \\
\hline Eudonia sudetica (Zeller, 1839) & & & & & & & & + & & & & & & \\
\hline \multicolumn{15}{|l|}{ Cybalomiinae } \\
\hline Hyperlais dulcinalis (Treitschke, 1835) & & & & + & & & & & & & & & & \\
\hline \multicolumn{15}{|l|}{ Odontiinae } \\
\hline Hellula undalis (Fabricius, 1781) & & & & & & & & & & & & & & + \\
\hline \multicolumn{15}{|l|}{ Pyraustinae } \\
\hline Ecpyrrhorrhoe rubiginalis (Hübner, 1796) & + & & & & & & & & & & & & & \\
\hline Paratalanta pandalis (Hübner, 1825) & & & + & & & & & & & & & & & \\
\hline
\end{tabular}




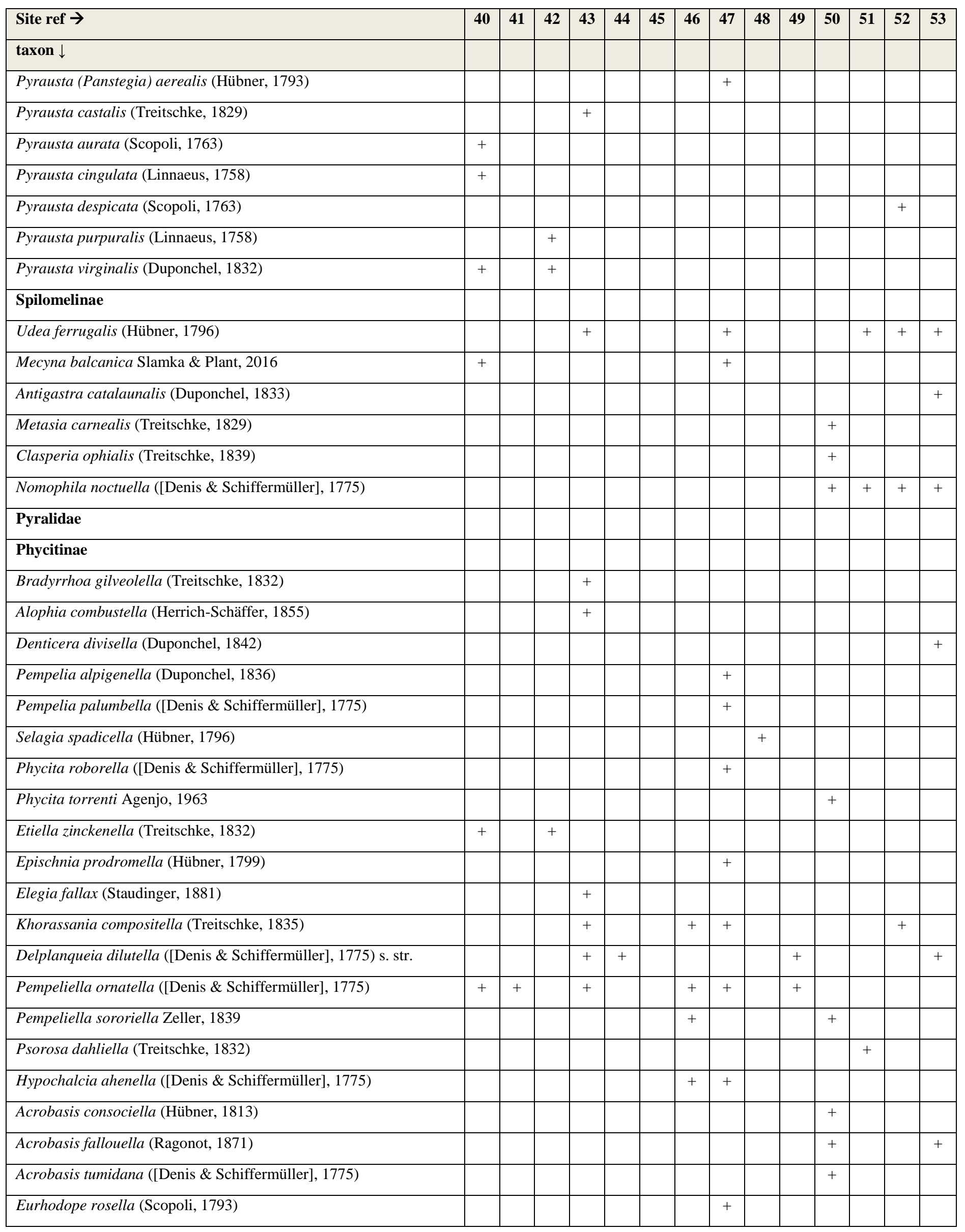




\begin{tabular}{|l|l|l|l|l|l|l|l|l|l|l|l|l|l|l|}
\hline Site ref $\rightarrow$ & $\mathbf{4 0}$ & $\mathbf{4 1}$ & $\mathbf{4 2}$ & $\mathbf{4 3}$ & $\mathbf{4 4}$ & $\mathbf{4 5}$ & $\mathbf{4 6}$ & $\mathbf{4 7}$ & $\mathbf{4 8}$ & $\mathbf{4 9}$ & $\mathbf{5 0}$ & $\mathbf{5 1}$ & $\mathbf{5 2}$ & $\mathbf{5 3}$ \\
\hline taxon $\downarrow$ & + & & & & & & & & & & & & & \\
\hline Eurhodope cirrigerella (Zincken, 1818) & + & & & & & & & & & & & & & \\
\hline Metallosticha argyrogrammos (Zeller, 1847) & + & + & & & & & & + & & & + & & \\
\hline Ancylosis cinnamomella (Duponchel, 1836) & & & & & & & + & & & & & & \\
\hline Homoeosoma sinuella (Fabricius, 1794) & & & & & & & & & & + & & & \\
\hline Phycitodes inquinatella (Ragonot, 1887) & & & & & & & & & & + & & & + \\
\hline Ephestia woodiella Richards \& Thompson, 1932 & & & & & & & & + & & & & & & \\
\hline Cadra furcatella (Herrich-Schäffer, 1849) & + & & & & & & & & & & & & \\
\hline Ematheudes punctella (Treitschke, 1833) & & & & & & & & & & & & & \\
\hline Pyralinae & + & & & & & & & & & + & & & \\
\hline Endotricha flammealis ([Denis \& Schiffermüller], 1775) & & & & & & & & & & + & & & \\
\hline Synaphe punctalis (Fabricius, 1775) & & & & & & & & & + & & & & & \\
\hline Stemmatophora brunnealis (Treitschke, 1839) & + & & & & & & & & & & & & \\
\hline Stemmatophora combustalis (Fischer Von Röslerstamm, 1842) & + & & & & & & & & & \\
\hline Hypsopygia costalis (Fabricius, 1775) & & & & & & & & & & & & + & \\
\hline Hypsopygia glaucinalis (Linnaeus, 1758) & & & & & & & & & & & & + \\
\hline
\end{tabular}

The taxonomy of the Pyraloidea is in a state of fluidity, with much current research being undertaken. In the presentation of our results we follow, in broad terms, the nomenclature adopted by Slamka $(2006 ; 2008 ; 2013)$ and for groups not yet reviewed by him we follow Leraut $(2009 ; 2014)$. However, we have updated names where more recent data are available. For simplicity, we have adopted the sequence of subfamilies and species presented by Slamka (op. cit.) for the Crambidae and Leraut (2014) for the Pyralidae.

\section{DISCUSSION}

\section{General comments}

The pyraloid fauna of the Balkan Peninsula, apart from that of Bulgaria, is rather poorly known. Plant (2016) lists 224 Crambidae and 165 Pyralidae for Bulgaria, a total of 389 taxa. Rákosy, Goia \& Kovács (2003) recognised 391 species (217 Crambidae and 174 Pyralidae) within the Romanian fauna. Hungary, to the north, is not a part of the Balkan Peninsula, but it is of interest for comparative purposes to record that Pastorális (2010) lists 305 species (171 Crambidae and 134 Pyralidae). There seems to be no comprehensive inventory for Greece to the south; the only available list appears to be that within Karsholt \& Razowski (1996).

A major problem affecting faunal listings for Macedonia, Montenegro and southern Serbia is one of historical politics; these areas have been incorporated under the label of "Yugoslavia" as a single entity. For the former Republic of
Yugoslavia, Karsholt \& Razowski (1996) list 390 species (202 Crambidae and 188 Pyralidae), but in addition to the lack of distinction between the component territories, the area covered also includes Bosnia \& Herzegovina, Croatia and Slovenia.

As far as we are aware, the earliest comprehensive inventory of Pyraloidea for any part of the southern Balkans within the present study area is that which can be found in the work by Rebel \& Zerny ([1931] 1934). This important work addresses the fauna of Albania, Macedonia and Montenegro and lists 175 species in total, of which 113 belong in the present day Crambidae and 62 within Pyralidae sensu stricto. However, a measure of interpretation is required in transferring the data from this paper to a modern database, since it was published at a time when the national boundaries of countries within the Balkans followed different lines. For example, a significant number of records have, as their source locality, "Mazedonien: Alibotuš Pl." (usually credited to Drenowski). This refers to the Alibotush Mountain area, now called Slavyanka Mountain and firmly within the boundary of the modern-day Republic of Bulgaria.

Thurner (1941) lists a total of 182 pyraloid taxa from the Lake Ohrid area of Macedonia. Careful examination of synonymy and a consideration of taxonomic changes that have taken place in the intervening 78 years reduce this list by ten, leaving 172 valid species recorded by Thurner for Macedonia. Just over twenty years later Moucha (1963) reported several new lepidopteran species for Albania, including 7 Pyraloidea, collected in 1959, but contemporary works on all of these areas are sparse. Misja (1987) reported 54 species of Tortricoidea and Pyraloidea of which 11 were tortricids and 43 were Pyraloidea 
and of these, 16 pyrales were stated to be new to the Albanian fauna. Mihajlović et al (1991) listed 77 species for Durmitor in Montenegro. Most recently, Beshkov (1994; 1996) reported on several species of migratory Lepidoptera in Albania and Macedonia mentioning three species in each year (a total of five species altogether). The earlier of the two papers included Palpita vitrealis (Rossi, 1794) from three localities in Albania a species claimed later, erroneously, as new to Albania by Płóciennik, Pawlikiewicz \& Pabis (2009). Karsholt \& Razowski (1996) summarised available published data at that date and listed 185 taxa (98 Crambidae and 87 Pyralidae) for Albania.

We are not aware of any existing formal checklist of Serbian Pyraloidea, although one is in long-term preparation (Dejan Stojanović, pers. comm., 2017). Stojanović \& Radaković (2016) mention 45 Crambidae and 29 Pyralidae from the Đerdap National Park. Jakšić (2016a), reported on the monitoring of Lepidoptera at the Zvezdarska Forest Nature Monument in
Belgrade, listing 11 species of Pyraloidea; neither of these studies affect our current, casual definition of southern Serbia.

Leraut $(2009,2014)$ provided distribution maps for most species (and text for the remainder) in which the different Balkan countries were distinguished. However, some maps contain errors with regard to other parts of Europe and since original data sources are not cited it is not possible to know if all of the maps are accurate in relation to the Balkan Peninsula. Accurate distribution maps were provided by Slamka (2006; 2008; 2013), but this series of books is incomplete and so a total number of species is not available. Most recently, Jakšić (2016) provided a tentative list of Serbian microlepidoptera based on a search of published sources only and lists 146 species of Crambidae and 82 Pyralidae - a total of 228 taxa. This work provides the most up to date inventory, but it omits unpublished data so that the list is still incomplete. Table 8 summarises the numbers of pyraloid taxa already known (published) from the different territories of the Balkans (and Hungary).

Table 8. Summary of published data on recorded pyraloid taxa in the Balkans and Hungary.

\begin{tabular}{|l|c|c|c|}
\hline \multirow{2}{*}{ Region } & \multicolumn{3}{|c|}{ Number of } \\
\cline { 2 - 4 } & Crambidae & Pyralidae & all Pyraloidea \\
\hline Hungary (Pastorális, 2010) & 171 & 134 & 305 \\
\hline Romania (Rákosy, Goia \& Kovács, 2003) & 217 & 174 & 391 \\
\hline Bulgaria (Plant, 2016) & 224 & 169 & 389 \\
\hline F. R. Yugoslavia (Karsholt \& Razowski, 1996) & 202 & 188 & 390 \\
\hline Greece (Karsholt \& Razowski, 1996) & 192 & 165 & 357 \\
\hline Albania (Karsholt \& Razowski, 1996) & 98 & 87 & 185 \\
\hline Serbia (Jakšić, 2016) & 146 & 82 & 228 \\
\hline
\end{tabular}

Plant (2016) used a free statistical program (EstimateS) to predict that the Bulgarian fauna might rise to 420 species, but concluded that the rate of recruitment would be so low that this process would take a further 150 years of survey: thus the Bulgarian list of pyrales can be regarded as complete. With that in mind, the close similarity between the total numbers for Romania, Bulgaria and Yugoslavia is interesting and suggests that a fauna of just below 400 species might reasonably be expected for a country the size of Serbia.
Albania, though doubtless much under-recorded, occupies a significantly smaller area of land and has a less varied range of biotopes within its boundary; it is perhaps less likely to achieve a comparable total.

This present work adds 29 species to the Serbian fauna and 37 to that of Albania, so that these lists rise to 257 and 222 respectively (Table 9). A further species (3 Crambidae and 2 Pyralidae) appear to be new to the published fauna of Macedonia.

Table 8. Summary of published data on recorded pyraloid taxa in the Balkans and Hungary.

\begin{tabular}{|c|c|c|c|c|c|c|c|c|c|}
\hline \multirow[t]{3}{*}{ Region } & \multicolumn{9}{|c|}{ Number of species of } \\
\hline & \multicolumn{3}{|c|}{ Crambidae } & \multicolumn{3}{|c|}{ Pyralidae } & \multicolumn{3}{|c|}{ all Pyraloidea } \\
\hline & was & added here & now & was & added here & now & was & added here & now \\
\hline Serbia & 146 & 8 & 154 & 82 & 21 & 103 & 228 & 29 & 257 \\
\hline Albania & 98 & 20 & 117 & 87 & 17 & 105 & 185 & 37 & 222 \\
\hline Macedonia & $?$ & 3 & $?$ & $?$ & 2 & $?$ & $?$ & 5 & $?$ \\
\hline
\end{tabular}


Comments on selected species

\section{CRAMBIDAE}

CRAMBINAE

Crambus ericella (Hübner, 1813)

According to the distribution shown at the Fauna Europaea website (https://fauna-eu.org/cdm_dataportal/taxon/33bedfd95f66-4f05-8182-4b1ef2f9b348), accessed 3 September 2017, this moth is unrecorded from the entire of the Balkan Peninsula ("Yugoslavia", Greece and Bulgaria), although it is shown for Romania and for Hungary and Austria to the north. This might reflect the low level of recording effort here, although we can confirm the apparent absence from Bulgaria during the years 2002 to 2016 and so perhaps it is genuinely rare here. We found it only at Valjevo (site 17) in Serbia on 14 June 2017, where it was common in tall, open grassland growing between the edge of a Pinus woodland and an associated forest track that was dominated by a ruderal flora. Apparently new to the Serbian fauna.

Angustalius malacellus (Duponchel, 1836)

This is one of the smaller members of the Crambinae and appears to be extremely local, across the Mediterranean region from Spain to Greece and further east on Cyprus. Confusion is possible with Mesocrambus candiellus (Herrich-Schäffer, 1848) which is also present in the former Yugoslavia; the genitalia are distinctive in both sexes. Several females came to light at Valjevo (site 17) in Serbia on 14 June 2017 and there were 3 females in the sample from Albania in the Vukopoles River Gorge (site 34) on 25 September 2016. Apparently new to the Albanian fauna.

Agriphila tolli (Bleszyński, 1952)

This is a widespread and abundant "grass moth" present throughout eastern Europe, including the Balkans, as far north as Austria, Hungary and Romania. It appears to replace Agriphila geniculea (Haworth, 1811) in the south- east, although Slamka 2008) reports both species present in Austria, Hungary and Italy. All records of A. geniculea from any of the Balkan countries, at least in the south, are potentially misidentified examples of $A$. tolli; new material must be critically examined and voucher specimens that support older records should be located and checked.

Pediasia aridella (Thunberg, 1788)

This is a surprising record, but is confirmed by critical examination of the genitalia of specimens of both sexes from the Berat region of Albania in the Vukopoles River Gorge (site 34) on 25 September 2016. It is a species of central and northern parts of Europe and is extremely local everywhere south of Hungary. The moth also persists on the Black Sea coast in Bulgaria, at a site very close to the Romanian border (Plant, 2016). Apparently new to the Albanian fauna.

\section{SCOPARIINAE}

Scoparia basistrigalis Knaggs, 1866

Members of the genera Scoparia Haworth and Eudonia Billberg are hopelessly under-recorded across the whole of Europe and the Balkans present no exception. With experience, fresh examples of some species can be named with confidence, but examination of genitalia is often necessary, especially with worn material. Although it occurs in both lowland and montane biotopes, $S$. basistrigalis is most often encountered in woodland areas. We record it now from sites $14,17,18 \& 21$ in Serbia and sites $24 \& 26$ in Albania. Apparently new to the Serbian fauna. Apparently new to the Albanian fauna.

Scoparia perplexella (Zeller, 1839)

This is a species that is apparently endemic to the Balkan Peninsula, with known reports from Bosnia \& Herzegovina, Croatia, Macedonia and Greece, so it is not especially surprising that it is also present in Serbia. It is one of the larger "scops" and flies from the end of May to early July as single generation. We record it at sites 7 and 19. Apparently new to the Serbian fauna. Eudonia sudetica (Zeller, 1839)

Most published distribution maps suggest that this species does not extend as far south, in Europe, as the Balkan and Italian Peninsulas. The maps at the Fauna Europaea website concur, but are not fully up to date; they show this species "absent" (as opposed to "no data"), from adjacent Bulgaria, in spite of it having been recorded here in recent years (Plant, 2016). The present survey showed that this species is also present throughout the southern Balkans, with records here from three sites in Serbia, two in Albania and one in Macedonia. Nuss (2005) informs that it is associated with native Picea forest in low montane sites and with Pinus cembra and/or Juniperus spp. in upper montane areas. (above 500 metres). Apparently new to the Serbian fauna. Apparently new to the Albanian fauna. Apparently new to the Macedonian fauna.

\section{CYBALOMIINAE}

Hyperlais dulcinalis (Treitschke, 1835)

This rather attractive moth is known in Europe from Italy, Croatia, Hungary, Romania, Bulgaria, Macedonia and Greece, extending eastwards to the Russian territory of Sarepta. Its absence from the other countries of the Balkan Peninsula perhaps reflects under-recording. We found it in light traps at several sites in Serbia, in the Veles Region of Macedonia and in Albania near Bistricë (Delvina region) and above Drenovë (Korçë region). Apparently new to the Albanian fauna.

\section{ODONTIINAE}

Cynaeda gigantea Staudinger, 1880

Two male examples were recognised by genitalia dissection of "greasy" specimens from the Korçë region of Albania, above Kloçë, near the radio mast (site 28) on 4 July 2016. In Europe, this moth occurs in the Balkan Peninsula and the Black seaboard zone. Apparently new to the Albanian fauna. 


\section{GLAPHYRIINAE}

Hellula undalis (Fabricius, 1781)

This species is resident in the African and Asian continents and European examples are regarded as primary immigrants. We caught examples in light traps in Albania (site 31) in late September and Macedonia (site 53) in mid October. Apparently new to the Albanian fauna. Apparently new to the Macedonian fauna.

Hydriris ornatalis (Duponchel, 1832)

As with the preceding species, this is a primary immigrant in Europe to the Mediterranean Basin only. As a migrant, it does not, apparently, extend as far north as Hellula undalis. It is also potential resident in the southern areas of Spain (Barry Goater, pers. Comm.) whilst Corley (2015) gives several localities in Portugal, in two broods. We recorded two males at light in the Berat region of Albania, in the Vukopoles River Gorge (site 34) on 25 September 2016. Apparently new to the Albanian fauna.

\section{EVERGESTINAE}

Evergestis mundalis (Guenée, 1854)

Separation of this species from the rather more widespread E. aenealis (Denis \& Schiffermüller, 1775) is usually based on the colour of the scales adorning the vertex of the head of the adult moth, but it is easy for a measure of complacency to cloud scientific judgement. Greasy specimens of E. aenealis frequently appear to have a vertex that is concolorous with the rest of the body whilst examination of the genitalia suggests that some examples of $E$. mundalis may include a few yellowish scales on the vertex. Examination of the genitalia is advised in all cases of doubt and in this present study all identifications reported have relied on that technique. We record males and females from Serbia in the Preševo district, above Trnava (site 14) at an altitude of 800 metres and from Albania in the Korçë region, above Drenovë (site 29) at 1050 metres, both in early July 2016. Apparently new to the Serbian fauna. Apparently new to the Albanian fauna.

Evergestis politalis ([Denis \& Schiffermüller], 1775)

The absence of this species from most of the former Yugoslavia in published distribution maps is surprising. It could possibly be misidentified in flight for the broadly similar $E$. limbata (Linnaeus, 1767), although this too is lacking from most of the area under study. We recorded E. politalis from Bistricë , in the Delvina region of Albania (site 26) in early June. Apparently new to the Albanian fauna.

\section{Evergestis serratalis (Staudinger, 1870)}

Leraut (2012) refers to the distribution and status of this distinctive species as, simply, "Balkans, Crimea. Highly local". We record it here from two sites in the Korçë region of Albania (sites 31 and 37), both in late September and at Vardar-Pčinja in Macedonia (site 51) in mid October. It is probably fairly widespread in low and high montane habitats throughout the area under study. Apparently new to the Albanian fauna.

\section{PYRAUSTINAE}

Paratalanta pandalis (Hübner, 1825)

This is a species that is widespread and common in northern and central areas of Europe; it is far less frequent in the south. It is known from Albania and also from Bulgaria to the east. This present work records it now from Macedonia, in the Demir Kapija Gorge (site 42) and Serbia at Mt. Starac (site 16) and Mt. Šljivovički Vis (site 23). Apparently new to the Serbian fauna. Apparently new to the Macedonian fauna.

Achyra nudalis (Hübner, 1796)

Recorded at two sites in Albania, in the Delvinë region at Bistricë on 7 June (site 26) and above Stan-Karbunarë in the Lushnjë region on 11 August (site 36), suggesting that there are at least two generations of adults per year. Elsewhere in Europe, the larvae are reported on Camphorosma spp., Echium spp., Chenopodium spp., Amaranthus spp and Beta spp. The European distribution is circum-Mediterranean, but also extends inland away from any maritime influence in Bulgaria and then eastwards across Asiatic Turkey to the Caucasus and so there is potential for this moth to be found in Serbia.

Ostrinia nubilalis (Hübner, 1796)

The taxonomy of this apparently widespread and common European moth is confused - not least because different authors have applied conflicting names to various supposedly separate taxa. One taxon within this complex is known colloquially as the "European Corn Borer" and is a commercial pest species. However, another taxon, which some regard as a separate species and others only as an ecotype, appears to thrive on Artemisia as a larval pabulum. Consideration of this problem is beyond the scope of this present paper, but it should be noted that by reference to the male genitalia drawings presented in Slamka (2013), site 31, in Albania, sampled on 11 August 2016, produced both nubilalis nubilalis [= maysalis auctorum] (Slamka Fig. 78) and nubilalis scapulalis (Slamka Fig. 79) [= nubilalis nubilalis auctorm]. All other sites in the results Tables refer to nubilalis nubilalis [= maysalis auctorum] (Slamka Fig. 78).

\section{SPILOMELINAE}

\section{Udea numeralis (Hübner, 1796)}

Although apparently more frequent in western Europe, the range of this species extends along the Mediterranean coastline via Italy to Greece and Asiatic Turkey, so that it is unsurprising that we have now recorded it in Albania at Bistricë (site 26). Apparently new to the Albanian fauna.

Udea prunalis ([Denis \& Schiffermüller], 1775)

A history of under-recording is likely to explain the apparent absence, until now, of this species in Albania. We recorded it above Kloçë, near the radio mast, in the Korçë region (site 28). Apparently new to the Albanian fauna.

\section{Pleuroptya balteata (Fabricius, 1798)}

This is a species that is associated with the southern areas of Europe along the whole of the Mediterranean seaboard then extending eastwards across Asiatic Turkey and into Syria, Iran 
and adjacent territories. It is previously known from Serbia and Macedonia and we now report it from Albania at the Devolli Gorge in the Korçë region (site 24) and the Vukopoles River Gorge in the Berat region on two occasions (sites 33 \& 34). Apparently new to the Albanian fauna.

Mecyna balcanica Slamka \& Plant, 2016

This is the undescribed species number 144 in the recent work by Slamka (2013). Separation from $M$. lutealis (Duponchel) requires examination of genitalia in both sexes and it is probable that older reports of "lutealis" from south-eastern Europe are referable to this taxon (see Slamka \& Plant, 2016 for discussion and a recent distribution map). Consequently, old records of $M$. lutealis must be regarded as "unconfirmed" until such time as a voucher specimen has been located and dissected. The known distribution includes the Carpathian Mountains in northern Hungary and south-west Bulgaria as well as the mountainous regions of the former Yugoslavia; the type locality is Croatia, and the series of paratypes include material from both Montenegro and Macedonia. Further east, there are reports from Turkey and from the Caucasus. We record it here at numerous sites in Serbia, Albania, Macedonia and Montenegro between 586 and 1740 metres altitude where it is common and flies in July. It is likely to be widespread throughout the whole Balkan Peninsula. Apparently new to the Serbian fauna. Apparently new to the Albanian fauna.

Mecyna flavalis ([Denis \& Schiffermüller], 1775)

Although not a rare species, this is far more often encountered in Central Europe, with populations in the Balkans appearing to be scattered and sometimes isolated. Although usually recognisable with ease, worn specimens may resemble $M$. balcanica whilst bright specimens of the latter may on occasion be misidentified as the present species. Examination of genitalia is urged in all cases until the true distribution pattern has become clear. Apparently new to the Albanian fauna.

Mecyna subsequalis (Herrich-Schäffer, 1851)

This is a species of Turkey eastwards to Transcaucasia, extending southwards to the Lebanon and northwards around the Black Sea before extending back, westwards, only as far west as the Crimean Peninsula. In Europe, it is known from one site in the extreme south-west of Bulgaria (Plant, 2016), Greece and Crete. The discovery of males, conforming to ssp. subsequalis (Herrich-Schäffer), at two sites in Albania, on the coast at Palasë, near Dhërmi, altitude 274 metres (site 25) and in the Berat region in the Vukopoles River Gorge at 217 metres altitude (site 34) is a surprise. New to the Albanian fauna.

Mecyna trinalis ([Denis \& Schiffermüller], 1775)

A generally widespread and not uncommon species of xerothermic habitats in southern Europe. The apparent absence of past Serbian records for this somewhat distinctive species is likely to be a measure of the degree to which the country is under-recorded. We record it here at site 14 (Serbia) and site 29
(Albania), both in early July. Apparently new to the Serbian fauna.

Antigastra catalaunalis (Duponchel, 1833)

This is, essentially, a tropical species that is also established in the Mediterranean basin. Elsewhere in Europe it is recognised as a primary immigrant. It is unclear if populations along the Adriatic seaboard represent established residents or if they depend upon occasional immigration for their longer-term maintenance, but the examples reported in the present survey, from the Ionian coast of Albania on 17 October 2016 (site 38) and the Vardar River Valley of Macedonia on 20 October 2016 (site 53) were all caught in association with other species that are known to migrate, such as Udea ferrugalis (Hübner, 1796) and Nomophila noctuella ([Denis \& Schiffermüller], 1775). Apparently new to the Albanian fauna.

Spoladea recurvalis (Fabricius, 1775)

A native of tropical and subtropical regions, migrating north in late summer and perhaps with temporary residency in the extreme south of the Iberian peninsula, this distinctive species was, until fairly recently, known to European lepidopterologists as an immigrant to the western half of the continent. In recent years however, it has been recorded in the east, including southwest Bulgaria in 2009. The discovery of presumably immigrant examples in the samples from the Albanian coast between Butrint and Sarandë (site 39) on 18 October 2016 supports the notion that the global movement pattern has been altered Reasons for this are obscure, but climate change is an obvious candidate. Apparently new to the Albanian fauna.

Metasia gigantalis (Staudinger, 1871)

The drawing of the male genitalia at Fig. 122a, page 480 in Leraut (2012), which purport to belong to M. carnealis are in fact wrongly identified and in reality depict $M$. gigantalis. Those of the female at Fig. 123a on page 481 appear to be correct. More detailed drawings are available in Slamka (2013). The species is known from Sicily, Crete, Cyprus and Asiatic Turkey as well as from Greece, where it seems widespread and Macedonia, whence it is reported only from the Lake Ohrid area (Thurner, 1938-1941). We record it here from the Ionian Coast of Albania near Ksamil, between Butrint and Sarandë in mid October 2016 (site 39). Apparently new to the Albanian fauna.

\section{PYRALIDAE}

\section{GALLERIINAE}

Lamoria anella ([Denis \& Schiffermüller], 1775)

A large and, when fresh, distinctive species that is found in most of Europe apart from the far north. It is likely to be overlooked in many places. We recorded it on the coast near Dhërmi (site 25) and at Bistricë (site 26), both in Albania, in early June 2016. Apparently new to the Albanian fauna.

\section{PHYCITINAE}

Bradyrrhoa gilveolella (Treitschke, 1832)

This is very much an eastern species in Europe, from Sicily (but apparently not Italy) and then from the Balkan Peninsula 
eastwards across the Middle East as far as Kyrgyzstan. Our records here from the Preševo district of Serbia, above Trnava on two dates (sites 13 and 14) must surely represent underrecording. We also note it from Macedonia in the Veles Region, above Sveti Ilija Monastery (site 43). Apparently new to the Serbian fauna.

Isauria dilucidella ([Denis \& Schiffermüller], 1775)

A widespread species throughout southern Europe; the absence of earlier records from much of the Balkans doubtless reflects a poor recording effort. We light-trapped examples at above Drenovë (site 30) on 9 August 2016, above StanKarbunarë (site 36) on 11 August 29016 and on the coast between Butrint and Sarandë (site 39) on 18 October 2016, all in Albania. Apparently new to the Albanian fauna.

Laodamia faecella (Zeller, 1839)

Existing European distribution maps suggest that this species is absent from the whole of the Balkans apart from Romania. However, it is widespread, if local, in Bulgaria (Plant, 2016). Jakšić (2016) lists it for the Serbian fauna and the present survey records three sites in Serbia (sites 5, 6 and 7) in July 2014. We also report it in the Lushnjë region of Albania, above Stan-Karbunarë (site 36) on 11 August 2016. Apparently new to the Albanian fauna.

Sciota hostilis (Stephens, 1834)

Published distribution maps suggest that this species is absent from most of the Balkans. However, it is present in Greece and European Turkey in the south and then north in Hungary and other countries to the west and east, so may simply be under-recorded. That said, we have only a single record, suggesting that it might be rare here, from Suva Planina, above Bojanine Vode (site 2). Apparently new to the Serbian fauna. Denticera divisella (Duponchel, 1842)

This is a fairly widespread and not uncommon moth of southern Europe, the range of which extends southwards to include Sub-Saharan Africa. The lack of records from Balkan countries until now is a reflection of poor recording coverage. We caught examples in Serbia in the Pirot region (site 5), at four localities in Albania (sites 30, 34, 38 and 39) and in Macedonia along the road to Klisura in the Vardar River Valley (site 53). Apparently new to the Serbian fauna. Apparently new to the Albanian fauna.

\section{Phycita coronatella (Guenée, 1845)}

The recent recognition of Phycita cryptica (see next species) has thrown past records of most Phycita species (other than roborella (D. \& S., 1775)) into disarray and existing distribution maps may not be reliable for this and some other Phycita species. We recorded examples in the Pirot region of Serbia and the Korçë region of Albania (sites 6, 22 \& 28). Apparently new to the Serbian fauna. Confirmed for the Albanian fauna.
Phycita cryptica Plant \& Slamka, 2016

As the specific epithet suggests, this species was "hidden" amongst other members of the genus; past records of several other species might refer to this newly recognised taxon (vide Plant \& Slamka, 2016, for a detailed discussion). During the present survey we found males only at Crni Vrh, near Pirot (site 22) on 2 September 2016. New to the Serbian fauna.

Phycita meliella (Mann, 1864)

The comments under $P$. coronatella apply. We found examples of both sexes at four sites in Serbia (sites 8, 13, 15 \& $16)$ in the present study. Apparently new to the Serbian fauna.

Phycita poteriella (Zeller, 1846)

The comments under $P$. coronatella apply. We found female examples in the trap samples from above Trnava in the Preševo district of Serbia (site 14) on 9 July 2016. Apparently new to the Serbian fauna.

Phycita torrenti Agenjo, 1963

For many decades, this species was thought to be endemic to the Iberian Peninsula, but that is not so. Confusion within the genus has been mentioned above; P. torrenti is in fact widespread in southern Europe (vide Plant \& Slamka, 2016). We record it here from above Trnava in Serbia (site 13) and the Treska Gorge, Macedonia (site 50) and expect it to be found in many other places. Apparently new to the Serbian fauna. New to the Macedonian fauna.

\section{Dioryctria simplicella Heinemann, 1863}

This is primarily a species of the northern parts of Europe and its appearance in the south therefore requires comment. It has been recorded in adjacent Bulgaria, but not prior to the year 2003 (Plant, 2016) and there is a possibility that it has extended its range southwards in recent years. Its presence in Serbia at Divčibare, Kaona (site 17) and Tornik Ribna (site 19) might, therefore, be a recent phenomenon. In the case of worn or damaged specimens correct identification requires examination of the male genitalia. Apparently new to the Serbian fauna.

Elegia fallax (Staudinger, 1881)

Separation of all worn examples of E. fallax from $E$. similella (Zincken, 1818) requires examination of genitalia (both sexes are distinct in that respect); the number of "surprises" encountered over several years where specimens named as one taxon by a supposed expert appear to have "evolved" into the other taxon during museum storage suggests that even freshlyemerged adults might benefit from such critical appraisal. Both species are likely to be widespread in the region. A third species, E. fallaximima Nel \& Mazel, 2012 is known from the Iberian Peninsula; so far, it has not been found elsewhere, but without dissection it would be overlooked. We found E. fallax in Albania near Strelçë village in the Devolli Gorge (site 24) on 5 June 2016 and in Macedonia above Sveti Ilija Monastery, Veles Region (site 43) on 11 May 2016. Apparently new to the Albanian fauna. 
Pterothrixidia rufella (Duponchel, 1836)

A widespread and common species in all areas. The very similar taxon Pterothrixidia squalidella (Eversmann, 1842), which was raised to full species rank by Leraut (2014), has not been found in the present study area.

Pima boisduvaliella (Guenée, 1845)

Although known from the Balkans, this is evidently a very localised species. We found it near Strelca in the Korçë region of Albania (site 24) in June and in the Berat region in the Vukopoles River Gorge between Ibrollara and Vale (site 34) in late September. Apparently new to the Albanian fauna.

Delplanqueia dilutella ([Denis \& Schiffermüller], 1775) s. str.

Leraut (2001), resurrected the split of Pempeliella dilutella into two segregate taxa - inscriptella (Duponchel, 1836) and dilutella D. \& S. sensu stricto. The move remains controversial, although probably correct. Characters of wing morphology are treated by some workers as important identification features, but examination of material from a wide geographical range across Europe suggests that these features may not be quite so clear cut. The male genitalia usually show significant differences in the size and shape of the single cornutus of the aedeagus, although intermediate examples have been encountered in Bulgaria. A small difference in the fully everted vesica of the male has been reported (Plant, 2016) and that character (Fig. 2) coincides $100 \%$ with the form of the cornutus in specimens from Serbia, Macedonia and Albania. Old records of "dilutella" must. of course, be treated as unreliable unless voucher specimens are located and critically examined. Consequently: Apparently new to the Serbian fauna. Apparently new to the Albanian fauna. Apparently new to the Macedonian fauna.

Delplanqueia inscriptella (Duponchel, 1836)

This segregate of $D$. dilutella is discussed above. Male examples, identified on the shape of the cornutus of the aedeagus and on the presence of a small diverticulum positioned at the inner angle of the vesica (Fig. 2), were found at Zvijezda, Savina Voda, near the Jabuka Pass on 16 July 2014 (site 7), above Trnava in the Preševo district on 31 May 2016 (site 11) and in the Svrljiški Timok river gorge, near Niševacon 4 August 2016 (site 15) - all in Serbia. Apparently new to the Serbian fauna. Pempeliella bulgarica Slamka \& Plant, 2016

This species was recognised as distinct very recently, and is known at present only from Bulgaria, Asiatic Turkey and a single site in Hungary (an historic record). Its presence in other Balkan countries was predicted (Slamka \& Plant, 2016a). It has been confused with $P$. sororiella Zeller, 1839, which is also common in the region; male and female genitalia are distinct and provide easy separation, but old reports of "sororiella" are to be treated as unconfirmed until voucher specimens are traced and dissected. The present survey records both sexes at the site near Trgovište, below Vražji Kamen in Serbia (site 8) and above Stan-Karbunarë in the Lushnjë region of Albania (site 36). New to the Serbian fauna. New to the Albanian fauna.
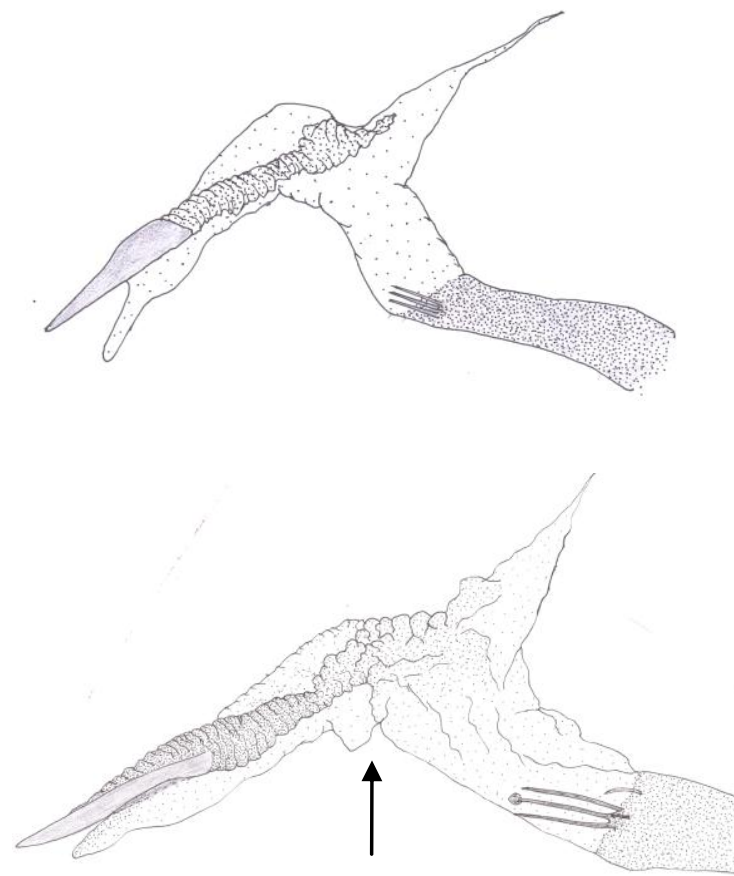

Figure 2. Everted vesica of the aedeagus of male Delplanqueia species: Top $-D$. dilutella sensu stricto; below $-D$. inscriptella. The arrow indicates the small additional diverticulum of inscriptella that is apparently absent from examples of dilutella. It is essential, in order to avoid false negatives, that the vesica is fully everted by careful use of a fine hypodermic syringe, or similar.

\section{Pempeliella sororiella Zeller, 1839}

We found this species at numerous sites in Serbia, Albania and Macedonia. Research undertaken by CWP on the distribution of Pempeliella bulgarica revealed, surprisingly, an absence of reports of $P$. sororiella from Serbia (vide map in Slamka \& Plant, 2016). Apparently new to the Serbian fauna. Oxybia transversella (Duponchel, 1836)

A fairly distinctive species when fresh, found throughout the southern part of Europe and eastwards across the Russian territories. We found it on three sites in Serbia and three more in Albania and expect that it will be added to the lists for most sites if they are properly surveyed. Apparently new to the Albanian fauna.

Psorosa nucleolella (Möschler, 1866)

Separation of this species from $P$. mediterranella is possible only through examination of the genitalia. Both taxa are present in Bulgaria, but whilst $P$. nucleolella was considered likely to be widespread there was only a single report of $P$. mediterranella (Plant, 2016). This latter species is prevalent in the western part of the Mediterranean Basin, but where the two meet is unclear and there is a high probability that they may overlap. Our single record of P. nucleolella in the present survey is from the coast, between Butrint \& Saranda, near Ksamil (site 39) on 18 September 2016. Apparently new to the Albanian fauna. 
Acrobasis advenella (Zincken, 1818)

A surprising omission from the Serbian fauna, until now where we report it from Pirot, above Kamenolom Kitka towards Crni Vrh at 870 metres altitude (site 5). Apparently new to the Serbian fauna.

Acrobasis consociella (Hübner, 1813)

Another species that is likely to prove to be widespread if properly looked for, since it is generally common across most of Europe. We found it at several sites in Serbia and Macedonia and at three localities in Albania (near Dhërmi on the cost, near Bistrice in the Delvina region and in the Vukopoles River Gorge between Ibrollara and Vale, Berat region. Apparently new to the Albanian fauna.

Acrobasis fallouella (Ragonot, 1871)

This species replaces A. glaucella Staudinger, 1859 in the east of Europe; males of the latter species are immediately recognised by the lack of a scale tuft at the base of the antenna (the "knot-horn"). This is present in A. fallouella males (Table 10). All existing records of A. glaucella are considered to be misidentifications of A. fallouella and the species Acrobasis glaucella is hereby formally deleted from the faunal lists for Serbia, Montenegro, Macedonia and Albania. A. fallouella is recorded by us at several sites in Serbia, Macedonia and Albania. Apparently new to the Serbian fauna. Apparently new to the Albanian fauna. Apparently new to the Macedonian fauna.

Table 10. Presence or absence of a "horn" of scales at the antennal base in European males of Acrobasis ZELLER, 1839.

\begin{tabular}{|c|c|c|}
\hline \multicolumn{3}{|c|}{ Antennal "thorn" in males of species of Acrobasis } \\
\hline Present & Absent & \\
\hline centunculella & advenella & \multirow{7}{*}{$\begin{array}{l}\text { Present or probably } \\
\text { present in the } \\
\text { current study area }\end{array}$} \\
\hline consociella & dulcella & \\
\hline fallouella & legatea & \\
\hline Oblique & marmorea & \\
\hline obtusella & sodalella & \\
\hline repandana & suavella & \\
\hline tumidana & - & \\
\hline bithynella & getuliella & \multirow{2}{*}{$\begin{array}{l}\text { Not yet found and probably } \\
\text { not expected in the current } \\
\text { study area }\end{array}$} \\
\hline porphyrella & glaucella & \\
\hline
\end{tabular}

Acrobasis legatea (Haworth, 1811)

As with others of this genus, the lack of past reports is a probable consequence of under-recording. We found it in both Serbia and Albania - in the in the Korçë region of the latter country, above Kloçë, near the radio mast, (site 28). Apparently new to the Albanian fauna.

Acrobasis repandana (Fabricius, 1798)

Another overlooked species, found by us on the Albanian coast at Palasë, near Dhërmi. Further survey is likely to show this species to be widespread and common in the region. Apparently new to the Albanian fauna.

Episcythrastis tabidella (Mann, 1864)

This somewhat nondescript, grey phycitine moth was found by us at two sites in Serbia, near Trgovište (site 8) and at Turski Grob (site 16), but is likely to be more or less widespread across the area under examination. It is a common member of the fauna in adjacent Bulgaria. Apparently new to the Serbian fauna.

Eurhodope cirrigerella (Zincken, 1818)

Listed as Kyra cirrigerella in many identification guides, this is a species of calcareous grassland habitats where the larval foodplants (Knautia and Scabiosa) are plentiful. Although both of the present records are from light traps, the adult insect can also be netted during the daytime. We found it near Babin Kal in the Bela Palanka district of Serbia (site 4) on 3 July 2014 and in the Pivska Planina Mountains of Montenegro at Trsa village, near Kulići, on17 July 2014. Apparently new to the Serbian fauna.

Euzopherodes charlottae (Rebel, 1914)

This small moth is relatively distinctive, though has been confused with an extreme form of E. vapidella (Mann, 1857) in which the median fascia is atypically emphasised. In Europe, $E$. charlottae occurs in Austria, Hungary and throughout the Balkans, extending east to the Middle East, There are also a very few reports from the Mediterranean area of France. In the present survey we recorded it once only, at Turski Grob, above the Pčinja river valley (site 16) on 5 August 2016. Apparently new to the Serbian fauna.

Phycitodes bentickella Pierce, 1937

Leraut (2002) recognised this species, as P. eliseannae, as a cryptic sibling of $P$. lacteella (Rothschild, 1915), but later (Leraut, 2014) recognised it as synonymous with $P$. bentickella. Plant (2016) records a single example from Bulgaria, captured on 12 August 2011. The present survey records it near the Jabuka Pass at Savina Voda in Serbia (site 7) on 16 July 2014, but this is the only other Balkan record. New to the Serbian fauna. Ephestia woodiella Richards \& Thompson, 1932

Leraut (2002) revised the synonymy of this a widespread and common species, which is already reported throughout the area under review, but under a variety of synonyms including $E$. parasitella Staudinger, 1859 and E. unicolorella Staudinger, 1881. The true "parasitella" is found in Switzerland and Iberia as well as North Africa; the true "unicolorella" is noted from Turkey and Syria. The European taxon is now regarded as "woodiella" and it is to this taxon which all material seen by us can be referred. 
Cadra furcatella (Herrich-Schäffer, 1849)

This species extends from Portugal to Turkey, but is confined to the southern half of Europe. Under-recording in the region is doubtless the reason why it was considered to be absent from Serbia and Albania until now. We record it in Serbia at Suva Planina, above Bojanine Vode (site 2) on 2 July 2014 and in Albania in the Devolli Gorge, near Strelçë (site 24) on 5 June 2016. Apparently new to the Serbian fauna. Apparently new to the Albanian fauna.

\section{PYRALINAE}

Stemmatophora brunnealis (Treitschke, 1839)

A widespread and common species in most parts of Europe, particularly central and southern parts. We record it here from three sites in Serbia (sites, 16, 22 and 23) and one in Albania (site 36). Apparently new to the Albanian fauna.

Stemmatophora honestalis (Treitschke, 1829)

Central Europe and the Balkans are the main areas of population of this species in Europe and so absence from the existing Serbian list is surprising. We found it above Kamenolom Kitka towards Crni Vrh in the Pirot region on 4 July 2014 (site 5). Apparently new to the Serbian fauna.

\section{ACKNOWLEGMENTS}

Permits for fieldwork in protected areas within Serbia were kindly provided by the Ministry of Environment, Mining and Spatial Planning, Republic of Serbia, No. 353-01-834/2017-17, dated 11 May 2017. SB and AN would like to thank Branko \& Nikola Micevski (Republic of Macedonia, Skopje, Macedonian Entomological Society ENTOMAK) for collecting permits for the Republic of Macedonia and for financial support for two of their trips. All other aspects of this entire project were selffunded by the authors. Andrew Smith (Chelmsford, England) created the map at Figure 1 from initial artwork provided by CWP. Barry Goater (Chandlers Ford, England) read an early draft of this paper and made helpful comments which we have adopted.

\section{REFERENCES}

Beshkov, S. 1994. Migrant Lepidoptera species in Macedonia and Albania, 1993 (Lepidoptera). Atalanta, 25(3-4), pp. 461468.

Beshkov, S. 1996. Migrant Lepidoptera species in Albania and Macedonia in 1995. Atalanta, 27(3/4), pp. 535-543.

Corley, M.F.V. 2015. Lepidoptera of Continental Portugal. Privately published, Corley. Oxford: Faringdon.

Friese, G. 1967. Ergebnisse der Albanien-Expedition 1961 des Deutschen Entomologischen Institutes. 61. Beitrag. Verzeichnis albanischer Fundorte. Beiträge zur Entomologie, 17(3-4), pp. 405-434.

Jakšić, P. 2016. Tentative checklist of Serbian microlepidoptera. Ecologica Montenegrina. Podgorica, 7, pp. $33-258$
Jakšić, P. 2016. Doprinos poznavanju faune noćnih leptira (Insecta: Lepidoptera) spomenika prirode „Zvezdarska šuma“ $\mathrm{u}$ Beogradu / A contribution to the knowledge of the moths fauna (Insecta: Lepidoptera) of the Zvezdara forest nature monument. Zaštita prirode / Nature Conservation, 66(2), pp. 35-40. Beograd, In Serbian, English summary.

Jovanović, G. 2010. Srbija, auto atlas 1: 200 000.Beograd.

Karsholt, O., \& Razowski, J. 1996. The Lepidoptera of Europe A Distributional Checklist. Stenstrup: Apollo Books., pp. 1380.

Leraut, P. 2001. Contribution à l'étude des Phycites Paléarctiques [Lepidoptera, Pyralidae, Phycitinae]. Nouvelle Revue d'Entomologie: Paris, 23(2), pp. 129-141.

Leraut, P. 2002. Contribution à l'étude des Phycites Paléarctiques [Lepidoptera, Pyralidae, Phycitinae]. Nouvelle Revue d'Entomologie (N. S.), Paris, 19(2), pp. 141-177.

Leraut, P. 2009. Zygaenids \& pyralids. In Moths of Europe. NAP Editions. Volume 3.

Leraut, P. 2014. Pyralids 2. In Moths of Europe. NAP Editions. Volume 4.

Mihajlović, Lj., Zečević, M., \& Jakšić, P. 1991. Pyralidae. In G. Nonveiler Ed., Fauna Durmitora.Titograd: Crnogorska akademija nauka i umjetnosti., pp. 243-275. In Serbian, English summary.

Misja, K. 1987. Të Dhëna Paraprake për Flatralusporët e Vegjël (Microlepidoptera) të Vendit Tonë / Some data about the Lepidoptera of our Country. Bulletin I Shkëncave Natyrore, 3, pp. 61-65.

Moucha, J. 1963. Eine Lepidopterenausbeute aus Albanien. Entomologisches Nachrichtenblatt (N.F.), 10(1), pp. 5-7. (2): 12-16.

Nuss, M. 2005. Scopariinae. In B. Goater, M. Nuss, \& W. Speidel Eds., Pyraloidea 1: Crambidae: Acentropinae, Evergestinae, Heliothelinae, Schoenobiinae, Scopariinae). Microlepidoptera of Europe. Apollo Books. Volume 4.

Pastorális, G. 2010. A checklist of microlepidoptera (Lepidoptera) occurreing in the territory of Hungary (version 1. 4). e-Acta Naturalia Pannonica, 1(1), pp. 89-170.

Plant, C.W. 2016. An annotated, systematic, synonymic and distributional checklist of the Pyraloidea of Bulgaria. Neue Entomologische Nachrichten, 72, pp. 1-351.

Plant, C.W., \& Slamka, F. 2016. Re-examination and revision of Zeller's original concept of Phycita Metzneri (Zeller, 1846) and description of Phycita cryptica sp. nov. (Lepidoptera, Pyraloidea, Pyralidae, Phycitinae). Entomologist's Record and Journal of Variation, 128, pp. 28-40.

Płóciennik, M., Pawlikiewicz, P., \& Pabis, K. 2009. Palpita vitrealis (Rossi, 1794) and Lygephila craccae ([Denis \& Schiffermüller], 1775) - Lepidoptera new for the fauna of Albania. J. Ent. Res. Soc., 11(2), pp. 39-41.

Rákosy, L., Goia, M., \& Kovács, Z. 2003. Catolgul Lepidopterelor României - Verzeichnis der Schmetterlinge Rumänaiens.Cluj Napolca: Societatea Lepidopterologica Romana.

Rebel, H., \& Zerny, H. 1931. 1934. Die Lepidopterenfauna Albaniens (mit Berücksichtigung der Nachbargebiete). Denkschriften / Akademie der Wissenschaften in Wien, Mathematisch-Naturwissenschaftliche.Wien: Hölder-PichlerTempsky, A.-G., pp. 1-21. Klasse 103: 37-161, text-figs 1-10, pl. 1, figs1-21, 1 map. 
Slamka, F. 2006. Lepidoptera: Volume 1: Pyralinae, Galleriinae, Epipaschiinae. In Pyraloidea of Europe. Bratislava: Cathariinae \& Odontiinae.

Slamka, F. 2008. Lepidoptera. In Pyraloidea of Europe. Bratislava. Volume 2.

Slamka, F. 2013. Lepidoptera: Pyraustinae and Spilomelinae. In Pyraloidea of Europe.Bratislava. Volume 3.

Slamka, F., \& Plant, C.W. 1839. 2016a. Pempeliella bulgarica sp. nov. a new species closely related to Pempeliella sororiella (Zeller, (Pyraloidea, Pyralidae, Phycitinae) and some new synonymies. Entomologist's Record and Journal of Variation, 128, pp. 99-111.
Slamka, F., \& Plant, C.W. 1833. 2016b. Mecyna balcanica sp. nov. a closely related species to Mecyna flavalis ([Denis \& Schiffermüller], 1775) and M. lutealis (Duponchel, (Pyraloidea, Crambidae, Spilomelinae). Entomologist's Record and Journal of Variation, 128, pp. 137-145.

Stojanović, D.V., \& Radaković, N.Z. 2016. Microlepidoptera (Pyraloidea). In Fauna Lepidoptera Nacionalnog Parka Đerdap.Donji Milanovac - Novi Sad. In Serbian.

Thurner, J. 1938. Die Schmetterlinge der Ochrid-gegend in Mazedonien. Mitteilungen aus den Königl. Naturwissenschaftlichen Instituten in Sofia. Bulletin des Institutions Royales d'Histoire Naturelle. A. Sofia Bulgarie, 14, pp. 9-35. 1938-1941. 